ALAIN BRILLARD

\title{
Asymptotic behaviour of a viscoplastic bingham fluid in porous media with periodic structure
}

\author{
Annales de la faculté des sciences de Toulouse $5^{e}$ série, tome 10, \\ $\mathrm{n}^{\mathrm{o}} 1$ (1989), p. 37-64 \\ <http://www.numdam.org/item?id=AFST_1989_5_10_1_37_0>
}

(C) Université Paul Sabatier, 1989, tous droits réservés.

L'accès aux archives de la revue «Annales de la faculté des sciences de Toulouse » (http://picard.ups-tlse.fr/ annales/) implique l'accord avec les conditions générales d'utilisation (http://www.numdam.org/conditions). Toute utilisation commerciale ou impression systématique est constitutive d'une infraction pénale. Toute copie ou impression de ce fichier doit contenir la présente mention de copyright.

\section{Numdam}

Article numérisé dans le cadre du programme Numérisation de documents anciens mathématiques http://www.numdam.org/ 


\title{
Asymptotic behaviour of a viscoplastic bingham fluid in porous media with periodic structure
}

\author{
Alain Brillard ${ }^{(1)}$
}

Résume. - Un fluide de Bingham viscoplastique et incompressible circule lentement dans un milieux poreux $\Omega_{\varepsilon}$ contenant une répartition $\varepsilon-$ périodique d'inclusions identiques. On étudie le comportement asymptotique de la vitesse $\vec{u}_{\varepsilon}$ du fluide, solution de

$$
\begin{gathered}
\operatorname{Min}_{\left(H_{0}^{1}(\Omega)\right)^{N}}\left(\frac{1}{2} \int_{\Omega_{e}} \sum_{i j}\left(e_{i j}(\vec{u})\right)^{2} d x+g \int_{\Omega_{e}}\left(\sum_{i j}\left(e_{i j}(\vec{u})\right)^{2}\right)^{1 / 2} d x\right. \\
\left.+\delta_{V\left(\Omega_{e}\right)}(\vec{u})-\int_{\Omega_{e}} \vec{f} \cdot \vec{u} d x\right)
\end{gathered}
$$

lorsque le paramètre $\varepsilon$ tend vers 0 . Les lois limites de Brinkman et de DARCY sont obtenues par des méthodes d'épi-convergence.

Abstract - A viscoplastic Bingham fluid slowly flows in a porous medium $\Omega_{\varepsilon}$ containing an $\varepsilon$-periodic distribution of identical inclusions. We study the behaviour of the velocity $\vec{u}_{\varepsilon}$, solution of the minimization problem

$$
\begin{gathered}
\underset{\left(H_{0}^{1}(\Omega)\right)^{N}}{\operatorname{Min}}\left(\frac{1}{2} \int_{\Omega_{e}} \sum_{i j}\left(e_{i j}(\vec{u})\right)^{2} d x+g \int_{\Omega_{e}}\left(\sum_{i j}\left(e_{i j}(\vec{u})\right)^{2}\right)^{1 / 2} d x\right. \\
+\delta_{V\left(\Omega_{e}\right)}(\vec{u})-\int_{\Omega_{e}} \vec{f} \cdot \vec{u} d x
\end{gathered}
$$

when the parameter $\varepsilon$ goes to 0 . Epi-convergence methods are systematically used in order to derive the nonlinear BRINKMAN and DARCY limit laws, according to the size of the inclusions.

(1) Faculté des Sciences et Techniques, 4 Rue des Frères Lumière, 68093 Mulhouse Cedex, France 


\section{A. Brillard}

\section{Introduction}

Let $\Omega$ be a bounded open and smooth subset of $R^{N}(N=2$ or 3$)$ and $T$ be a smooth open subset of the unit ball $B(1)$ of $R^{N}$. The porous medium $\Omega_{\varepsilon}$ is equal to

$$
\Omega_{\varepsilon}=\Omega \backslash U_{i=1}^{I(\varepsilon)} \overline{T_{\varepsilon i}},
$$

where $T_{\varepsilon i}$ is a $r_{\varepsilon}$-homothetic of $T$ disposed at the center $x_{\varepsilon i}$ of the $i-$ th $\varepsilon-$ cell $Y_{\varepsilon i}$ of a rectangular $\varepsilon$-mesh covering $\Omega$ :

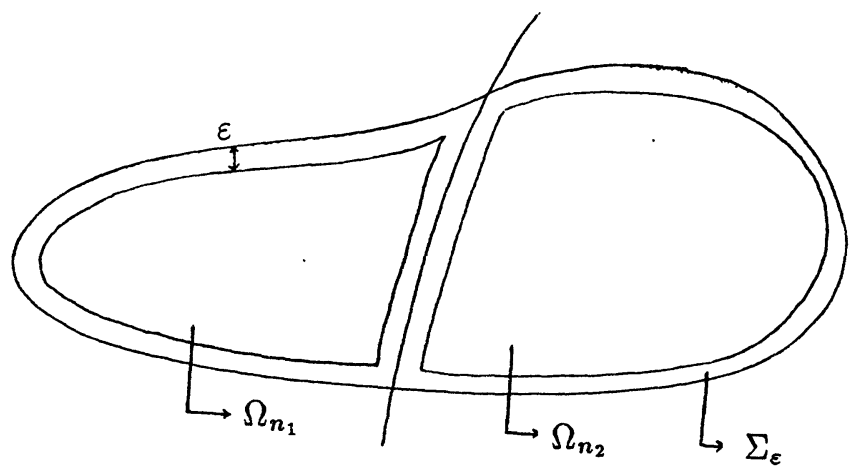

Figure 1

(notice that the number $I(\varepsilon)$ of solid inclusions $T_{\varepsilon i}$ is equivalent to Vol $\left.(\Omega) / \varepsilon^{N}\right)$. The problem we are interested in is the behaviour of the solution $\vec{u}_{\varepsilon}$ of the minimization problem [7]

$$
\operatorname{Min}_{\vec{u} \in\left(H_{0}^{1}(\Omega)\right)^{N}}\left(F^{\varepsilon}(\vec{u})-\int_{\Omega} \vec{f} \cdot \vec{u} d x\right),
$$

where $\vec{f}$ belongs to $\left(L^{2}(\Omega)\right)^{N}\left(\vec{f}\right.$ represents the exterior forces) and $F^{\varepsilon}$ is the functional defined on $\left(H_{0}^{1}(\Omega)\right)^{N}$ by

$$
F^{\varepsilon}(\vec{u})=\frac{1}{2} \int_{\Omega} \sum_{i j}\left(e_{i j}(\vec{u})\right)^{2} d x+g \int_{\Omega}\left(\sum_{i j}\left(e_{i j}(\vec{u})\right)^{2}\right)^{1 / 2} d x+\delta_{V\left(\Omega_{\varepsilon}\right)}(\vec{u}),
$$

$e_{i j}(\vec{u})$ being the deformation (symmetric) tensor : $\frac{1}{2}\left(D_{j} u_{i}+D_{i} u_{j}\right), g$ being a nonnegative real, $\delta_{V\left(\Omega_{e}\right)}$ denoting the indicator function of the closed subspace $V\left(\Omega_{\varepsilon}\right)$ of $\left(H_{0}^{1}\left(\Omega_{\varepsilon}\right)\right)^{N}$

$$
V\left(\Omega_{\varepsilon}\right)=\left\{\vec{u} \in\left(H_{0}^{1}\left(\Omega_{\varepsilon}\right)\right)^{N} / \operatorname{div} \vec{u}=\sum_{i=1}^{N} D_{i} u_{i}=0 \text { in } \Omega\right\} .
$$


The coefficient $g$ is equal to 0 for newtonian fluids and is strictly positive for Bingham fluids. The case $g=0$, has been studied in [2], [5], [9], [11], [12]. Let $P^{\varepsilon} \vec{u}_{\varepsilon}$ be the canonical extension of $\vec{u}_{\varepsilon}$ taking the value $\overrightarrow{0}$ on the inclusions (thanks to the no-slip condition on the boundary of the solid inclusions $T_{e i}$ ), then the asymptotic behaviour of the fluid is described in the following Theorem :

THEOREM 1.1.- (Brinkman's law : case $g=0)$ [5], [11]

Suppose that $: \lim _{\varepsilon \rightarrow 0}\left(r_{\varepsilon} / \varepsilon\right)=0$. Then the sequence $\left(P_{\varepsilon} \vec{u}_{\varepsilon}\right)_{\varepsilon}$ converges in the weak topology of $\left(H_{0}^{1}(\Omega)\right)^{N}$ to the solution $\vec{u}_{0}^{*}$ of

$$
\mid \begin{array}{ll}
-\Delta \vec{u}_{0}^{*}+M \vec{u}_{0}^{*}=-\operatorname{grad} p_{0}^{*}+\vec{f} & \text { in } \Omega, \\
\operatorname{div} \vec{u}_{0}^{*}=0 & \text { in } \Omega, \\
\vec{u}_{0}^{*}=\overrightarrow{0} & \text { on } \partial \Omega,
\end{array}
$$

where $M$ is the symmetric matrix given by:

for every $k, l$ in $\{1, \ldots, N\}$ :

$$
M_{k l}=\lim _{\varepsilon \rightarrow 0}\left(\frac{1}{\varepsilon^{N}} \int_{B(\varepsilon / 4) \backslash \bar{T}_{\varepsilon}} \sum_{i j}\left(e_{i j}\left(\vec{w}_{\varepsilon}^{k}\right) e_{i j}\left(\vec{w}_{\varepsilon}^{l}\right)\right) d x\right)
$$

$\vec{w}_{\varepsilon}^{k}$ being the solution of the local minimization problem :

$$
\begin{gathered}
\operatorname{Min} \int_{B(\varepsilon / 4) \backslash \bar{T}_{\epsilon}} \sum_{i j}\left(e_{i j}(\vec{w})\right)^{2} d x, \\
\mid \begin{array}{l}
\vec{w}=\vec{e}_{k} \text { on } \partial B(\varepsilon / 4) \\
\vec{w}=\overrightarrow{0} \text { on } \partial T_{\varepsilon} \\
\operatorname{div} \vec{w}=0 \text { in } B(\varepsilon / 4) \backslash \overline{T_{\varepsilon}} \\
\vec{w} \in\left(H^{1}(B(\varepsilon / 4))\right)^{N}
\end{array}
\end{gathered}
$$

$\left(\vec{e}_{k}\right.$ is the $k^{\text {th }}$ canonical vector of $\left.R^{N}\right)$.

Remark 1.2.-

1 - From the definition (3) of the matrix $M$, we infer the existence of a critical size $r_{\varepsilon}^{c}$ of the inclusions :

$$
r_{\varepsilon}^{c}=\mid \begin{array}{ll}
C \varepsilon^{3} & \text { if } N=3,\left(C \in R^{+*}\right) \\
\exp \left(-C \varepsilon^{-2}\right) & \text { if } N=2
\end{array}
$$

such that 


\section{A. Brillard}

a) if $\lim _{\varepsilon \rightarrow 0}\left(r_{\varepsilon} / r_{\varepsilon}^{c}\right)=0$, then the matrix $M$ is null and $\vec{u}_{0}^{*}$ is the solution of the Stokes problem in $\Omega$.

b) if $\lim _{\varepsilon \rightarrow 0}\left(r_{\varepsilon} / r_{\varepsilon}^{c}\right)=+\infty$, then $\vec{u}_{0}^{*}$ is equal to $\overrightarrow{0}$ (the fluid is at rest in $\Omega$ ).

c) if $\lim _{\varepsilon \rightarrow 0}\left(r_{\varepsilon} / r_{\varepsilon}^{c}\right)$ belongs to $R^{+*}$, then Brinkman's law contains a "strange term" $M \vec{u}_{0}^{*}$, which depends on the size and shape of the inclusions, through the capacity problem (4).

2 - The solution $\vec{u}_{0}^{*}$ of Brinkman's law is the solution of the following minimization problem :

$$
\underset{\left(H_{0}^{1}(\Omega)\right)^{N}}{\operatorname{Min}}\left(F^{*}(\vec{u})-\int_{\Omega} \vec{f} \cdot \vec{u} d x\right)
$$

where $F^{*}$ is the functional defined on $\left(H_{0}^{1}(\Omega)\right)^{N}$ by :

$$
F^{*}(\vec{u})=\frac{1}{2} \int_{\Omega} \sum_{i j}\left(e_{i j}(\vec{u})\right)^{2} d x+\frac{1}{2} \sum_{k l} \int_{\Omega} M_{k l} u_{k} u_{l} d x+\delta_{V(\Omega)}(\vec{u}) .
$$

In [5], thanks to the use of epi-convergence methods, have been given : the behaviour of the dissipation energy of the fluid, the convergence of the internal pressure (in some duality sense), the convergence of the solution of the Stokes evolution problem, the convergence of the spectra, some indications about first-order correctors.

In the next paragraph of the present work, the asymptotic behaviour of a Bingham fluid $(g>0)$, slowly flowing in the above-described porous medium, will be studied in the situation : $\lim _{\varepsilon \rightarrow 0}\left(r_{\varepsilon} / \varepsilon\right)=0$.

We will prove that the asymptotic velocity $\vec{u}_{0}$ of the Bingham fluid is the solution of the minimization problem : $\operatorname{Min}_{\vec{u} \in\left(H_{0}^{1}(\Omega)\right)^{N}}\left(F(\vec{u})-\int_{\Omega} \vec{f} \cdot \vec{u} d x\right)$, where $F$ is the functional defined on $\left(H_{0}^{1}(\Omega)\right)^{N}$ by

$$
\begin{aligned}
F(\vec{u}) & =\frac{1}{2} \int_{\Omega} \sum_{i j}\left(e_{i j}(\vec{u})\right)^{2} d x+\frac{1}{2} \sum_{k l} \int_{\Omega} M_{k l} u_{k} u_{l} d x \\
& +g \int_{\Omega}\left(\sum_{i j}\left(e_{i j}(\vec{u})\right)^{2}\right)^{1 / 2} d x+\delta_{V(\Omega)}(\vec{u}) .
\end{aligned}
$$

In this non-newtonian case, the limit functional $F$ still contains the "strange term" $\frac{1}{2} \sum_{k l} \int_{\Omega} M_{k l} u_{k} u_{l} d x$, where $M$ is the matrix given by (3). The nonnewtonian term $g \int_{\Omega}\left(\sum_{i j}\left(e_{i j}(\vec{u})\right)^{2}\right)^{1 / 2} d x$, appearing in the functional $F^{\varepsilon}(2)$ 
Asymptotic behaviour of a viscoplastic bingham fluid in porous media

and also in the limit functional $F$, does not alter this strange term. The proof of this result, presented in Theorem 2.1 is based on the two following
arguments :

- the explicit computation of the solution $\left(\vec{w}_{\varepsilon}^{k}, q_{\varepsilon}^{k}\right)$ of (4) when $T$ is equal to the unit ball $B(1)$ (see [5]), or the existence of pointwise estimates on this solution when $T$ is a smooth subset of $B(1)$ (see [11]). These computations or estimates imply that for every smooth divergence-free function $\vec{v}$ in $\left(H_{0}^{1}(\Omega)\right)^{N}$, there exists a sequence $\left(P^{\varepsilon} \vec{v}_{\varepsilon}^{0}\right)_{\varepsilon}$ converging to $\vec{v}$ in the weak topology of $\left(H_{0}^{1}(\Omega)\right)^{N}$ and such that :

$$
\begin{aligned}
& \lim _{\varepsilon \rightarrow 0} \frac{1}{2} \int_{\Omega_{\varepsilon}} \sum_{i j}\left(e_{i j}\left(\vec{v}_{\varepsilon}^{0}\right)\right)^{2} d x=F^{*}(\vec{v}), \\
& \lim _{\varepsilon \rightarrow 0} g \int_{\Omega_{\varepsilon}}\left(\sum_{i j}\left(e_{i j}\left(\vec{v}_{\varepsilon}^{0}\right)\right)^{2}\right)^{1 / 2} d x=g \int_{\Omega}\left(\sum_{i j}\left(e_{i j}(\vec{v})\right)^{2}\right)^{1 / 2} d x
\end{aligned}
$$

(see Proposition 2.3),

- the lower semicontinuity, with respect to the weak topology of $\left(H_{0}^{1}(\Omega)\right)^{N}$ of this non-newtonian term.

Then, we study the behaviour of the solution of the evolution problem associated to (1) (see Proposition 2.4).

In the last part of this paragraph, we consider the case of a coefficient $g$ depending on $\varepsilon$. We prove that, either the Bingham fluid has a linear asymptotic behaviour (when $\lim _{\varepsilon \rightarrow 0} g(\varepsilon)=0$ ), either the sequence $\left(P^{\varepsilon} \vec{u}_{\varepsilon}\right)_{\varepsilon}$ converges to $\overrightarrow{0}$ very fastly (when $\lim _{\varepsilon \rightarrow 0} g(\varepsilon)=+\infty$ ) (see Remark 2.5).

The particular case $r_{\varepsilon}=a \varepsilon(0<a<1 / 2)$ has been studied, for newtonian fluids, through the method of asymptotic expansions in $[2,9,12]$ and by means of epi-convergence methods in [5].

TheOREM 1.3. - (Darcy's law, case $g=0$ )

Suppose $r_{\varepsilon}=a \varepsilon(0<a<1 / 2)$. Then, the sequence $\left(\frac{1}{\varepsilon^{2}} P^{\varepsilon} \vec{u}_{\varepsilon}\right)_{\varepsilon}$ converges in the weak topology of $\left(L^{2}(\Omega)\right)^{N}$ to the function $\vec{u}_{1}^{*}$

$$
\begin{cases}\vec{u}_{1}^{*}=K\left(-\operatorname{grad} p_{1}^{*}+\vec{f}\right), & \\ \vec{u}_{1}^{*} \in H(\Omega)=\left\{u \in\left(L^{2}(\Omega)\right)^{N}\right. & / \operatorname{div} \vec{u}=0 \text { in } \Omega, \vec{u} \cdot \vec{n}=0 \text { on } \partial \Omega\}, \\ & (\vec{n} \text { is the outer normal to } \partial \Omega),\end{cases}
$$

where $K$ is the symmetric positive definite matrix given by: 


\section{A. Brillard}

for every $k, l$ in $\{1, \ldots, N\}$ :

$$
K_{k l}=\int_{Y \backslash \overline{a T}} \sum_{i j} e_{i j}\left(\bar{z}^{*}\right) e_{i j}\left(\bar{z}^{d}\right) d y=\int_{Y \backslash \overline{a T}} \bar{z}^{*} \cdot \vec{e}_{l} d y,
$$

$\vec{z}^{*}$ being the solution of the minimization problem:

Min

$$
\left(\frac{1}{2} \int_{Y \backslash \overline{a T}} \sum_{i j}\left(e_{i j}(\vec{z})\right)^{2} d y-\int_{Y \backslash \overline{a T}} \vec{z} \cdot \vec{e}_{k} d y\right) .
$$

$\vec{z} Y-$ periodic

$\vec{z}=\overrightarrow{0}$ on $a T$

$\operatorname{div} \vec{z}=0$ in $Y \backslash \overline{d a T}$

Remark 1.4. $-\vec{u}_{1}^{*}$ is the solution of the following minimization problem :

$$
\operatorname{Min}_{\vec{u} \in H(\Omega)}\left(\int_{\Omega} j(\vec{u}(x)) d x-\int_{\Omega} \vec{f} \cdot \vec{u} d x\right),
$$

where $j$ is the function defined from $R^{N}$ into $R$ by :

$$
\forall \eta \in R^{N}: j(\eta)=\frac{1}{2} \sum_{k l}\left(K^{-1}\right)_{k l} \eta_{k} \eta_{\ell} .
$$

An elementary computation shows that the function $j$ may be written in the following form :

$$
\begin{aligned}
\forall \eta \in R^{N}: j(\eta)=\operatorname{Min}_{\xi \in R^{N}} & \left(\frac{1}{2} \int_{Y \backslash \overline{a T}} \sum_{i j}\left(e_{i j}\left(\bar{z}_{\xi}^{*}\right)\right)^{2} d y+\right. \\
+ & \left.K^{-1} \eta \cdot\left(\eta-\int_{Y \backslash \overline{a T}} \vec{z}_{\xi}^{*} d y\right)\right),
\end{aligned}
$$

where $\vec{z}_{\xi}^{*}$ is the $Y$-periodic function equal to $\sum_{k} \xi_{k} \vec{z}^{*}$. Notice that $\vec{z}_{\xi}^{*}$ is the solution of :

$\operatorname{Min}$

$$
\left(\frac{1}{2} \int_{Y \backslash \overline{a T}} \sum_{i j}\left(e_{i j}(\vec{z})\right)^{2} d y-\int_{Y \backslash \overline{a T}} \vec{z} \cdot \xi d y\right) .
$$

$\vec{z} Y$ - periodic

$\vec{z}=0$ on $a T$

$\operatorname{div} \vec{z}=0$ in $Y \backslash \overline{a T}$ 
This case : $r_{\varepsilon}=a \varepsilon(0<a<1 / 2)$ leads, for a Bingham fluid (that is when $g$ is strictly positive and changed into $\varepsilon g$, through a dimension analysis of the coefficients [10]) to a nonlinear Darcy's law :

$$
\left\{\begin{array}{l}
\vec{u}_{1}=A_{0}\left(\vec{f}-\operatorname{grad} p_{1}\right), \\
\vec{u}_{1} \in H(\Omega)
\end{array}\right.
$$

where $A_{0}$ is the function from $R^{N}$ into $R^{N}$ equal to :

$$
A_{0}:\left(\begin{array}{cl}
R^{N} & \rightarrow R^{N} \\
\xi & \rightarrow \int_{Y \backslash \overline{a T}} \vec{z}_{\xi}(y) d y
\end{array}\right)
$$

$\vec{z}_{\xi}$ being the solution of the minimization problem :

$$
\begin{aligned}
& \text { Min }\left(\frac{1}{2} \int_{Y \backslash \overline{a T}} \sum_{i j}\left(e_{i j}(\vec{z})\right)^{2} d y+g \int_{Y \backslash \overline{a T}}\left(\sum_{i j}\left(e_{i j}(\vec{z})\right)^{2}\right)^{1 / 2} d y-\right. \\
& \vec{z} Y-\text { periodic } \\
& \begin{array}{l}
\vec{z}=\overrightarrow{0} \text { on } a T \\
\operatorname{div} \vec{z}=0 \text { in } Y
\end{array} \\
& \text { (compare to (6)). }
\end{aligned}
$$

This result has been partially proved in [10], by means of asymptotic expansions of the solution $\vec{u}_{\varepsilon}$ of (1). The purpose of the third part of the present work is to furnish a different proof of this nonlinear Darcy's law, by means of epi-convergence methods.

We shall first prove that $\left(\varepsilon^{-2} P^{\varepsilon} \vec{u}_{\varepsilon}\right)_{\varepsilon}$ is a bounded sequence in $\left(L^{2}(\Omega)\right)^{N}$. Noticing that $\varepsilon^{-2} P^{\varepsilon} \vec{u}_{\varepsilon}$ is the solution of a minimization problem :

$$
\operatorname{Min}_{\left(H_{0}^{1}(\Omega)\right)^{N}}\left(G^{\varepsilon}(\vec{u})-\int_{\Omega} \vec{f} \cdot \vec{u} d x\right),
$$

where

$$
G^{\varepsilon}(\vec{u})=\frac{\varepsilon^{2}}{2} \int_{\Omega} \sum_{i j}\left(e_{i j}(\vec{u})\right)^{2} d x+\varepsilon g \int_{\Omega}\left(\sum_{i j}\left(e_{i j}(\vec{u})\right)^{2}\right)^{1 / 2} d x+\delta_{V\left(\Omega_{\epsilon}\right)}(\vec{u}),
$$

the nonlinear Darcy's law will be derived from the computation of the epilimit $G$ of the sequence $\left(G^{\varepsilon}\right)_{\varepsilon}$ in the weak topology of $\left(L^{2}(\Omega)\right)^{N}$ : 


\section{A. Brillard}

$$
\begin{aligned}
& G(\vec{u})=\int_{\Omega} j_{0}(\vec{u}(x)) d x-\int_{\Omega} \vec{f} \cdot \vec{u} d x \quad \text { with } \\
& \forall \eta \in R^{N}: j_{0}(\eta)=\operatorname{Min}_{\xi \in R^{N}}\left(\frac{1}{2} \int_{Y \backslash \overline{a T}} \sum_{i j}\left(e_{i j}(\vec{z})\right)^{2} d y+\right. \\
& \quad+g \int_{Y \backslash \overline{a T}}\left(\sum_{i j}\left(e_{i j}\left(\vec{z}_{\xi}\right)\right)^{2}\right)^{1 / 2} d y+ \\
&\left.+A_{0}^{-1} \eta \cdot\left(\eta-\int_{Y \backslash \bar{a} \overline{z_{\xi}}} \vec{z}_{\xi} d y\right)\right) .
\end{aligned}
$$

The last part of this study deals with the convergence of the internal pressure of the Bingham fluid. Thanks to Tartar's idea [13], we shall prove the existence of an extension $\bar{p}_{\varepsilon}$ of the internal pressure $p_{\varepsilon}$ of the fluid, such that $\left(\bar{p}_{\varepsilon}\right)_{\varepsilon}$ converges in the strong topology of $L^{2}(\Omega) / R$, to a function $p_{2}$. We conjecture that this function $p_{2}$ is in fact equal to the limit pressure $p_{1}$, appearing in the nonlinear Darcy's limit law.

Let us now recall the main properties of epi-convergence, which will be used throughout this study.

\section{DEFINITION 1.5 [1], [6].-}

Let $(X, \tau)$ be a metric vector space and $\left(F^{\varepsilon}\right)_{\varepsilon}$ a sequence of functionals from $X$ into $\bar{R}$. Then, $\left(F^{\varepsilon}\right)_{\varepsilon}$ epi-converges to $F$, in the topology $\tau$, if

$$
\forall x \in X:\left(\tau-\liminf _{\varepsilon \rightarrow 0} F^{\varepsilon}\right)(x)=\left(\tau-\limsup _{\varepsilon \rightarrow 0} F^{\varepsilon}\right)(x)=F(x),
$$

where : $\quad\left(\tau-\liminf _{\varepsilon \rightarrow 0} F^{\varepsilon}\right)(x)=\operatorname{Min}_{\left(x_{\varepsilon}\right)_{\varepsilon}} \liminf _{\varepsilon \rightarrow 0} F^{\varepsilon}\left(x_{\varepsilon}\right)$,

$$
\left(\tau-\limsup _{\varepsilon \rightarrow 0} F^{\varepsilon}\right)(x)=\operatorname{Min}_{\left(x_{\varepsilon}\right)_{\varepsilon}} \limsup _{\varepsilon \rightarrow 0} F^{\varepsilon}\left(x_{\varepsilon}\right),
$$

$\left(x_{\varepsilon}\right)_{\varepsilon}$ being any sequence converging to $x$, in the topology $\tau$.

Equivalently, this equality is satisfied if the two following assertions are fulfilled

$$
\begin{gathered}
\text { for every } x \text { in } X, \text { there exists a sequence }\left(x_{\varepsilon}^{0}\right)_{\varepsilon} \\
\tau \text {-converging to } x, \text { such that } \limsup _{\varepsilon \rightarrow 0} F^{\varepsilon}\left(x_{\varepsilon}^{0}\right) \leq F(x), \\
\text { for every } x \text { in } X \text { and for every sequence }\left(x_{\varepsilon}\right)_{\varepsilon} \\
\tau \text {-converging to } x: \liminf _{\varepsilon \rightarrow 0} F^{\varepsilon}\left(x_{\varepsilon}\right) \geq F(x) .
\end{gathered}
$$

This variational convergence is well-fitted to the asymptotic analysis of minimization problems : 
Asymptotic behaviour of a viscoplastic bingham fluid in porous media

THEOREM 1.6 ([1] Theorem 1.10).-

Suppose that $\left(F^{\varepsilon}\right)_{\varepsilon}$ epi-converges to $F$, in the topology $\tau$ and let $\bar{x}_{\varepsilon}$ be an $o_{\varepsilon}$-minimizer of $F^{\varepsilon}$, that is : $F^{\varepsilon}\left(\bar{x}_{\varepsilon}\right) \leq \operatorname{Inf}_{x \in X} F^{\varepsilon}(x)+o_{\varepsilon}$, with $\lim _{\varepsilon \rightarrow 0} o_{\varepsilon}=0$. Suppose, moreover, that the sequence $\left(\bar{x}_{\varepsilon}\right)_{\varepsilon}$ is $\tau$-relatively compact.

Then, for every $\tau$-converging subsequence $\left(\overline{x_{\varepsilon^{\prime}}}\right)_{\varepsilon^{\prime}}$ of $\left(\overline{x_{\varepsilon}}\right)_{\varepsilon}$, with $\bar{x}=$ $\lim _{\varepsilon^{\prime} \rightarrow 0} \overline{x_{\varepsilon^{\prime}}}$ :

$$
F(\bar{x})=\operatorname{Min}_{X} F(x) .
$$

and moreover $\lim _{\varepsilon^{\prime} \rightarrow 0} F^{\varepsilon^{\prime}}\left(\bar{x}_{\varepsilon^{\prime}}\right)=F(\bar{x})$.

The next result shows the stability of epi-convergence, with respect to continuous perturbations.

Proposition 1.7 ([1] Theorem 2.15.).-

Suppose that $\left(F^{\varepsilon}\right)_{\varepsilon}$ epi-converges to $F$, in the topology $\tau$ and $G$ is continuous for this topology $\tau$. Then $\left(F^{\varepsilon}+G\right)_{\varepsilon}$ epi-converges to $F+G$ in the topology $\tau$.

NOTATIONS :

$L^{2}(\Omega), H_{0}^{1}(\Omega)$ are the classical function spaces, while $\left(L^{2}(\Omega)\right)^{N}$ and $\left(H_{0}^{1}(\Omega)\right)^{N}$ consist of vector-valued functions whose components belong to $L^{2}(\Omega)$ or $H_{0}^{1}(\Omega)$.

$\mathcal{V}(\Omega)$ is the space of smooth divergence-free functions :

$$
\mathcal{V}(\Omega)=\left\{\vec{u} \in C_{0}^{\infty}(\Omega) / \operatorname{div} \vec{u}=\sum_{i=1}^{N} D_{i} u_{i}=0 \text { in } \Omega\right\},
$$

$\mathcal{V}(\Omega)$ is dense in $V(\Omega)$ for the topology of $\left(H_{0}^{1}(\Omega)\right)^{N}$ and in $H(\Omega)$ for the topology of $\left(L^{2}(\Omega)\right)^{N}$, where

$V(\Omega)=\left\{\vec{u} \in\left(H_{0}^{1}(\Omega)\right)^{N} / \operatorname{div} \vec{u}=0\right.$ in $\left.\Omega\right\}$, $H(\Omega)=\left\{\vec{u} \in\left(L^{2}(\Omega)\right)^{N} / \operatorname{div} \vec{u}=0\right.$ in $\Omega$ and $\vec{u} \cdot \vec{n}=0$ on $\partial \Omega$, where $\vec{n}$ is the outer normal to the (smooth) boundary $\partial \Omega\}$.

$\delta_{A}$ is the indicator function of the set $A: \delta_{A}(x)=\mid \begin{array}{ll}0 & \text { if } x \text { belongs to } A, \\ +\infty & \text { elsewhere. }\end{array}$ $\chi_{A}$ is the characteristic function of the set $A: \chi_{A}(x)=\mid \begin{aligned} & 1 \text { if } x \text { belongs to } A \text {, } \\ & 0 \text { elsewhere. }\end{aligned}$ 


\section{A. Brillard}

For every $z$ in $L^{1}(Y)\left(Y\right.$ is the unit cell $\left[-\frac{1}{2}, \frac{1}{2}\right]^{N}$ of $\left.R^{N}\right), \widetilde{z}$ denotes the mean value of $z$ on $Y$

$$
\widetilde{z}=\int_{Y} z(y) d y \text {. }
$$

A function $z$ defined on $Y$ is called $Y$-periodic if it takes the same values on the opposite sides of $Y$.

\section{Asymptotic behaviour of the solution $\overrightarrow{\mathbf{u}}_{\varepsilon}$ of (1) when g $>0$ and $\lim _{\varepsilon \rightarrow 0}\left(\mathbf{r}_{\varepsilon} / \varepsilon\right)=0$}

$P^{\varepsilon}$ denotes the canonical extension operator from $\left(H_{0}^{1}\left(\Omega_{\varepsilon}\right)\right)^{N}$ into $\left(H_{0}^{1}(\Omega)\right)^{N}$ :

$$
P^{\varepsilon} \vec{u}=\mid \begin{aligned}
& \vec{u} \text { on } \Omega_{\varepsilon} \\
& \overrightarrow{0} \text { on } \bigcup_{i} T_{\varepsilon i}
\end{aligned}
$$

From the strict positivity of $g$, Poincare and Korn's inequalities in $\Omega$ [7], one first deduces from (1), that $\left(P^{\varepsilon} \vec{u}_{\varepsilon}\right)_{\varepsilon}$ is bounded in $\left(H_{0}^{1}(\Omega)\right)^{N}$.

\section{THEOREM 2.1.}

The sequence $\left(P^{\varepsilon} \vec{u}_{\varepsilon}\right)_{\varepsilon}$ (canonical extension of the solution of (1)) converges in the weak topology of $\left(H_{0}^{1}(\Omega)\right)^{N}$ to the solution $\vec{u}_{0}$ of :

$$
\operatorname{Min}_{\vec{u} \in\left(H_{0}^{1}(\Omega)\right)^{N}}\left(F(\vec{u})-\int_{\Omega} \vec{f} \cdot \vec{u} d x\right),
$$

where $F$ is the functional defined by

$$
\begin{aligned}
F(\vec{u})=\frac{1}{2} \int_{\Omega} \sum_{i j}\left(e_{i j}(\vec{u})\right)^{2} d x & +\frac{1}{2} \sum_{k l} \int_{\Omega} M_{k l} u_{k} u_{l} d x+ \\
& +g \int_{\Omega}\left(\sum_{i j}\left(e_{i j}(\vec{u})^{2}\right)\right)^{1 / 2} d x+\delta_{V(\Omega)}(\vec{u}),
\end{aligned}
$$

$M$ being the symmetric matrix given by (3).

Moreover, the dissipation energy of the fluid :

$$
\frac{1}{2} \int_{\Omega_{\varepsilon}} \sum_{i j}\left(e_{i j}\left(\vec{u}_{\varepsilon}\right)\right)^{2} d x+g \int_{\Omega_{\varepsilon}}\left(\sum_{i j}\left(e_{i j}\left(\vec{u}_{\varepsilon}\right)\right)^{2}\right)^{1 / 2} d x
$$


Asymptotic behaviour of a viscoplastic bingham fluid in porous media

converges to the energy of the asymptotic fluid :

$\frac{1}{2} \int_{\Omega} \sum_{i j}\left(e_{i j}\left(\vec{u}_{0}\right)\right)^{2} d x+\frac{1}{2} \int_{\Omega} \sum_{k l} M_{k l}\left(\vec{u}_{0}\right)_{k}\left(\vec{u}_{0}\right)_{l} d x+g \int_{\Omega}\left(\sum_{i j}\left(e_{i j}\left(\vec{u}_{0}\right)\right)^{2}\right)^{1 / 2} d x$

This Theorem 2.1 is a straightforward consequence of the following result,

THEOREM 2.2 .

The sequence $\left(F^{\varepsilon}\right)_{\varepsilon}$ of functionals defined by (2) epi-converges to the functional $F$ defined by (9), in the weak topology of $\left(H_{0}^{1}(\Omega)\right)^{N}$,

through theorem 1.6, since

- the functional $F$ is convex and strictly coercive on $\left(H_{0}^{1}(\Omega)\right)^{N}$ (hence the minimizer of $F$ is unique and the whole sequence $\left(P^{\varepsilon} \vec{u}_{\varepsilon}\right)_{\varepsilon}$ converges to $\left.\vec{u}_{0}\right)$, - the functional : $\vec{u} \longrightarrow \int_{\Omega} \vec{f} \cdot \vec{u} d x$, is continuous for the weak topology of $\left(H_{0}^{1}(\Omega)\right)^{N}$.

Proof of Theorem 2.2:

Noticing that $V(\Omega)$ is a closed subspace of $\left(H_{0}^{1}(\Omega)\right)^{N}$, for the weak topology of this space, one proves that for every $\vec{v}$ in $\left(H_{0}^{1}(\Omega)\right)^{N}$, which - is not divergence-free in $\Omega$ :

$\left(\left(w-\left(H_{0}^{1}(\Omega)\right)^{N}\right)-\limsup _{\varepsilon \rightarrow 0} F^{\varepsilon}\right)(\vec{v})=\left(\left(w-\left(H_{0}^{1}(\Omega)\right)^{N}\right)-\liminf _{\varepsilon \rightarrow 0} F^{\varepsilon}\right)(\vec{v})=+\infty$.

In order to prove Theorem 2.2, one has to verify the assertions (7) and (8) which take the special form :

For every $\vec{v}$ in $V(\Omega)$, there exists a sequence $\left(P^{\varepsilon} \vec{v}_{\varepsilon}^{0}\right)_{\varepsilon}$, converging in the weak topology of $\left(H_{0}^{1}(\Omega)\right)^{N}$ to $\vec{v}$, with $\vec{v}_{\varepsilon}^{0}$ in $V\left(\Omega_{\varepsilon}\right)$, such that

$$
\limsup _{\varepsilon \rightarrow 0} F^{\varepsilon}\left(\vec{v}_{\varepsilon}^{0}\right) \leq F(\vec{v})
$$

for every $\vec{v}$ in $V(\Omega)$ and for every sequence $\left(P^{\varepsilon} \vec{v}_{\varepsilon}\right)_{\varepsilon}$ converging to $\vec{v}$ in the weak topology of $\left(H_{0}^{1}(\Omega)\right)^{N}$, with $\vec{v}_{\varepsilon}$ in $V\left(\Omega_{\varepsilon}\right)$ :

$$
\liminf _{\varepsilon \rightarrow 0} F^{\varepsilon}\left(\vec{v}_{\varepsilon}\right) \geq F(\vec{v}) .
$$

Suppose first that $\alpha_{N}$ is finite, with

$$
\alpha_{N}=\mid \begin{array}{ll}
\lim _{\varepsilon \rightarrow 0}\left(r_{\varepsilon} \varepsilon^{-3}\right) & \text { if } N=3 \\
\lim _{\varepsilon \rightarrow 0} \frac{\varepsilon^{-2}}{\left|\log r_{\varepsilon}\right|} & \text { if } N=2
\end{array}
$$




\section{A. Brillard}

The verification of (10) and (11) is a consequence of the following Proposition, the technical proof of which being postponed in Appendix 1 :

Proposition 2.3. - Suppose that $\vec{v}$ belongs to $\mathcal{V}(\Omega)$ and $\alpha_{N}$ is finite. Then, there exists a function $\vec{v}_{\varepsilon}^{0}$ in $V\left(\Omega_{\varepsilon}\right)$ satisfying

a) $\left(P^{\varepsilon} \vec{v}_{\varepsilon}^{0}\right)_{\varepsilon}$ converges to $\vec{v}$ in the weak topology of $\left(H_{0}^{1}(\Omega)\right)^{N}$.

b) $\left(\int_{\Omega_{\varepsilon}} \sum_{i j}\left(e_{i j}\left(\vec{v}_{\varepsilon}^{0}\right)\right)^{2} d x\right)_{\varepsilon_{2}}$ converges to

$\int_{\Omega} \sum_{i j}\left(e_{i j}(\vec{v})\right)^{2} d x+\sum_{k l} \int_{\Omega}^{\varepsilon_{2}} M_{k l} v_{k} v_{l} d x$.

c) there exists a constant $C$ independant of $\vec{v}$ such that for every $\vec{w}$ in $V(\Omega)$ and for every sequence $\left(P^{\varepsilon} \vec{w}_{\varepsilon}\right)_{\varepsilon}$ converging to $\vec{w}$, in the weak topology of $\left(H_{0}^{1}(\Omega)\right)^{N}$, with $\vec{w}_{\varepsilon}$ in $V\left(\Omega_{\varepsilon}\right)$ :

$$
\begin{aligned}
\liminf _{\varepsilon \rightarrow 0} \int_{\Omega_{\varepsilon}} \sum_{i j} e_{i j}\left(\vec{v}_{\varepsilon}^{0}\right) e_{i j}\left(\vec{w}_{\varepsilon}\right) d x & \geq \int_{\Omega} \sum_{i j} e_{i j}(\vec{v}) e_{i j}(\vec{w}) d x \\
& -C\|\vec{w}\|_{\left(L^{2}(\Omega)\right)^{N}}\|\vec{v}\|_{\left(L^{2}(\Omega)\right)^{N}}
\end{aligned}
$$

d) $\left(\int_{\Omega_{\varepsilon}}\left(\sum_{i j}\left(e_{i j}\left(\vec{v}_{\varepsilon}^{0}\right)\right)^{2}\right)^{1 / 2} d x\right)_{\varepsilon}$ converges to $\int_{\Omega}\left(\sum_{i j}\left(e_{i j}(\vec{v})\right)^{2}\right)^{1 / 2} d x$.

Let us prove (10) for a smooth divergence-free function $\vec{v}$ in $\mathcal{V}(\Omega)$. Proposition $2.3 \mathrm{a}), \mathrm{b})$ and d) imply the existence of $\left(P^{\varepsilon} \vec{v}_{\varepsilon}^{0}\right)_{\varepsilon}$ converging to $\vec{v}$ in the weak topology of $\left(H_{0}^{1}(\Omega)\right)^{N}$ and such that :

$$
\lim _{\varepsilon \rightarrow 0} F^{\varepsilon}\left(\vec{v}_{\varepsilon}^{0}\right)=F(\vec{v})
$$

Therefore, (10) is proved for a smooth divergence-free function $\vec{v}$ in $\mathcal{V}(\Omega)$.

If $\vec{v}$ belongs to $V(\Omega)$, there exists a sequence $\left(\vec{v}_{n}\right)_{n}$ of smooth divergencefree functions in $\mathcal{V}(\Omega)$, converging to $\vec{v}$ in the strong topology of $\left(H_{0}^{1}(\Omega)\right)^{N}$. For every $n$, Proposition 2.3 guarantees the existence of $\left(P^{\varepsilon}\left(\vec{v}_{n}\right)_{\varepsilon}^{0}\right)_{\varepsilon}$ converging to $\vec{v}_{n}$, in the weak topology of $\left(H_{0}^{1}(\Omega)\right)^{N}$, such that :

$$
\lim _{\varepsilon \rightarrow 0} F^{\varepsilon}\left(\left(\vec{v}_{n}\right)_{\varepsilon}^{0}\right)=F\left(\vec{v}_{n}\right)
$$

Hence

$$
\limsup _{n \rightarrow+\infty} \limsup _{\varepsilon \rightarrow 0} F^{\varepsilon}\left(\left(\vec{v}_{n}\right)_{\varepsilon}^{0}\right) \leq F(\vec{v})
$$


Asymptotic behaviour of a viscoplastic bingham fluid in porous media

thanks to the continuity of $F$, with respect to the strong topology of $\left(H_{0}^{1}(\Omega)\right)^{N}$. Using the diagonalization argument of Corollary 1.16. [1], one derives the existence of a subsequence $(n(\varepsilon))_{\varepsilon}$ growing to $+\infty$ such that :

$$
\limsup _{\varepsilon \rightarrow 0} F^{\varepsilon}\left(\left(\vec{v}_{n(\varepsilon)}\right)_{\varepsilon}^{0}\right) \leq F(\vec{v}),
$$

and $\left(\left(P^{\varepsilon}\left(\vec{v}_{n(\varepsilon)}\right)_{\varepsilon}^{0}\right)_{\varepsilon}\right.$ converges to $\vec{v}$ in the weak topology of $\left(H_{0}^{1}(\Omega)\right)^{N}$. Take $\vec{v}_{\varepsilon}^{0}=\left(\vec{v}_{n(\varepsilon)}\right)_{\varepsilon}^{0}:(10)$ is proved for $\vec{v}$ in $V(\Omega)$ (and $\alpha_{N}$ finite).

Let us now prove (11) when $\alpha_{N}$ is finite : take $\vec{v}$ in $V(\Omega),\left(P^{\varepsilon} \vec{v}_{\varepsilon}\right)_{\varepsilon}$ (resp. $\left.\left(\vec{v}_{n}\right)_{n}\right)$ converging to $\vec{v}$ in the weak (resp. strong) topology of $\left(H_{0}^{1}(\Omega)\right)^{N}, \vec{v}_{\varepsilon}$ in $V\left(\Omega_{\varepsilon}\right), \vec{v}_{n}$ in $\mathcal{V}(\Omega)$.

$$
\begin{aligned}
\liminf _{\varepsilon \rightarrow 0} F^{\varepsilon}\left(\vec{v}_{\varepsilon}\right) \geq & \frac{1}{2} \liminf _{\varepsilon \rightarrow 0} \int_{\Omega_{\varepsilon}} \sum_{i j}\left(e_{i j}\left(\vec{v}_{\varepsilon}\right)\right)^{2} d x+ \\
& +g \liminf _{\varepsilon \rightarrow 0} \int_{\Omega_{\varepsilon}}\left(\sum_{i j}\left(e_{i j}\left(\vec{v}_{\varepsilon}\right)\right)^{2}\right)^{1 / 2} d x \\
\geq & \frac{1}{2} \liminf _{\varepsilon \rightarrow 0} \int_{\Omega_{\varepsilon}} \sum_{i j}\left(e_{i j}\left(\left(\vec{v}_{n}\right)_{\varepsilon}^{0}\right)\right)^{2} d x+ \\
& +\liminf _{\varepsilon \rightarrow 0} \int_{\Omega_{\varepsilon}} \sum_{i j} e_{i j}\left(\left(\vec{v}_{n}\right)_{\varepsilon}^{0}\right) e_{i j}\left(\vec{v}_{\varepsilon}-\left(\vec{v}_{n}\right)_{\varepsilon}^{0}\right) d x+ \\
& +g \liminf _{\varepsilon \rightarrow 0} \int_{\Omega_{\varepsilon}}\left(\sum_{i j}\left(e_{i j}\left(\vec{v}_{\varepsilon}\right)\right)^{2}\right)^{1 / 2} d x .
\end{aligned}
$$

Thanks to Proposition 2. 3. b) and c), one obtains

$$
\begin{aligned}
& \liminf _{\varepsilon \rightarrow 0} F^{\varepsilon}\left(\vec{v}_{\varepsilon}\right) \geq \frac{1}{2} \int_{\Omega} \sum_{i j}\left(e_{i j}\left(\vec{v}_{n}\right)\right)^{2} d x+\frac{1}{2} \sum_{k l} \int_{\Omega} M_{k l}\left(\vec{v}_{n}\right)_{k}\left(\vec{v}_{n}\right)_{l} d x+ \\
& \quad+\int_{\Omega} \sum_{i j} e_{i j}\left(\vec{v}_{n}\right) e_{i j}\left(\vec{v}-\vec{v}_{n}\right) d x-C\left\|\vec{v}_{n}\right\|_{\left(L^{2}(\Omega)\right)^{N}}\left\|\vec{v}-\vec{v}_{n}\right\|_{\left(L^{2}(\Omega)\right)^{N}+} \\
& \quad+g \liminf _{\varepsilon \rightarrow 0} \int_{\Omega_{\varepsilon}}\left(\sum_{i j}\left(e_{i j}\left(\vec{v}_{\varepsilon}\right)\right)^{2}\right)^{1 / 2} d x .
\end{aligned}
$$

Moreover the functional

$$
\vec{v} \rightarrow \int_{\Omega}\left(\sum_{i j}\left(e_{i j}(\vec{v})\right)^{2}\right)^{1 / 2} d x
$$

is convex and continuous for the strong topology of $\left(H_{0}^{1}(\Omega)\right)^{N}$, hence lower semicontinuous for the weak topology of this space. 


\section{A. Brillard}

Let $n$ go to $+\infty$, and use the property of $\left(\vec{v}_{n}\right)_{n}:(11)$ is proved.

Suppose finally that $\alpha_{N}$ is equal to $+\infty$.

Observe first that for every $\vec{v}$ in $V(\Omega)$ and for every $\alpha$ in $R^{+*}$ :

$$
F^{\varepsilon}(\vec{v}) \geq F_{\alpha}^{\varepsilon}(v),
$$

where $F_{\alpha}^{\varepsilon}$ is the functional $F^{\varepsilon}$ corresponding to the case $\alpha_{N}=\alpha$ and $T=B(1)$. From the properties of the matrix $M$, one immediately deduces

$$
\begin{aligned}
\left(\left(w-\left(H_{0}^{1}(\Omega)\right)^{N}\right)-\limsup _{\varepsilon \rightarrow 0} F^{\varepsilon}\right)(\vec{v}) & =\left(\left(w-\left(H_{0}^{1}(\Omega)\right)^{N}\right)-\liminf _{\varepsilon \rightarrow 0} F^{\varepsilon}\right)(\vec{v})= \\
& =\mid \begin{array}{l}
0 \text { if } \vec{v}=\overrightarrow{0}(\text { a.e. }) \text { in } \Omega, \\
+\infty \text { if } \vec{v} \text { is not equal to } \overrightarrow{0} \text { in } \Omega .
\end{array}
\end{aligned}
$$

This ends the proof of Theorem 2.2.

Let us conclude this paragraph, giving the convergence of the solution of the evolution problems.

Proposition 2.4.- Take $T_{0}$ in $R^{+*},\left(\vec{f}_{\varepsilon}\right)_{\varepsilon}$ (resp. $\left.\left(\vec{x}_{\varepsilon}\right)_{\varepsilon}\right)$ a sequence converging to $\vec{f}$ in $L^{2}\left(0, T_{0} ;\left(L^{2}(\Omega)\right)^{N}\right)$ (resp. to $\vec{x}$ in $\left.\left(L^{2}(\Omega)\right)^{N}\right)$. Then the sequence $\left(P^{\varepsilon} \vec{u}_{\varepsilon}(., .)\right)_{\varepsilon}$ of extensions of the solution $\vec{u}_{\varepsilon}$ of

$$
\mid \begin{aligned}
& \left.\frac{\partial \vec{u}_{\varepsilon}}{\partial t}+\partial F^{\varepsilon}\left(\vec{u}_{\varepsilon}\right) \ni \vec{f}_{\varepsilon} i n\right] 0, T_{0}\left[\times \Omega_{\varepsilon},\right. \\
& \vec{u}_{\varepsilon}(., t) \in V\left(\Omega_{\varepsilon}\right) \text { for } t>0 \\
& \vec{u}_{\varepsilon}(x, 0)=\vec{x}_{\varepsilon}
\end{aligned}
$$

converges in the strong topology of $L^{2}\left(0, T_{0} ;\left(L^{2}(\Omega)\right)^{N}\right)$ to the solution $\vec{u}_{0}$ of

$$
\mid \begin{aligned}
& \frac{\partial \vec{u}_{0}}{\partial t}+\partial F\left(\vec{u}_{0}\right) \ni \vec{f}_{0}, \\
& \vec{u}_{0}(., t) \in V(\Omega) \text { for } t>0, \\
& \vec{u}_{0}(x, 0)=\vec{x} .
\end{aligned}
$$

Moreover

$$
\int_{0}^{T_{0}} t\left|\frac{\partial \vec{u}_{\varepsilon}}{\partial t}-\frac{\partial \vec{u}_{0}}{\partial t}\right|^{2} d t \underset{\varepsilon \rightarrow 0}{\longrightarrow} 0
$$

Proof of Proposition 2.4.

See [1] Theorem 3.74.

Remark 2.5. - In this section, we have supposed $g$ to be a constant. Let us now consider the case of a coefficient $g$ equal to some function $g(\varepsilon)$, that is

$$
F^{\varepsilon}(\vec{u})=\frac{1}{2} \int_{\Omega} \sum_{i j}\left(e_{i j}(\vec{u})\right)^{2} d x+g(\varepsilon) \int_{\Omega}\left(\sum_{i j}\left(e_{i j}(\vec{u})\right)^{2}\right)^{1 / 2} d x+\delta_{V\left(\Omega_{\varepsilon}\right)}(\vec{u}) .
$$


Asymptotic behaviour of a viscoplastic bingham fluid in porous media

1 - If $\lim _{\varepsilon \rightarrow 0} g(\varepsilon)=0$, then one immediately proves, with the arguments developped in the proof of Theorem 2.2, that $\left(F^{\varepsilon}\right)_{\varepsilon}$ epi-converges in the weak topology of $\left(H_{0}^{1}(\Omega)\right)^{N}$, to the functional $F^{*}$ defined by $(5)$. In this case, the (weakly) non-newtonian fluid has a linear asymptotic behaviour. 2 - If $\lim _{\varepsilon \rightarrow 0} g(\varepsilon)=+\infty$ and if $\vec{f}$ belongs to $\left(C^{0}(\bar{\Omega})\right)^{N}$, for example, then $\left((g(\varepsilon))^{p} P^{\varepsilon} \vec{u}_{\varepsilon}\right)_{\varepsilon}$ converges to $\overrightarrow{0}$, in the strong topology of $\left(H_{0}^{1}(\Omega)\right)^{N}$ for every integer p. Indeed, observe first that :

$$
\begin{aligned}
\int_{\Omega} \sum_{i j}\left(e_{i j}\left(P^{\varepsilon} \vec{u}_{\varepsilon}\right)\right)^{2} d x & +g(\varepsilon) \int_{\Omega}\left(\sum_{i j}\left(e_{i j}\left(P^{\varepsilon} \vec{u}_{\varepsilon}\right)\right)^{2}\right)^{1 / 2} d x= \\
& =\int_{\Omega} \vec{f} \cdot P^{\varepsilon} \vec{u}_{\varepsilon} d x .
\end{aligned}
$$

Since $\left(P^{\varepsilon} \vec{u}_{\varepsilon}\right)_{\varepsilon}$ is bounded in $\left(H_{0}^{1}(\Omega)\right)^{N}$, one proves that

$$
\left(\int_{\Omega}\left(\sum_{i j}\left(e_{i j}\left(P^{\varepsilon} \vec{u}_{\varepsilon}\right)\right)^{2}\right)^{1 / 2} d x\right)_{\varepsilon} \text { converges to 0. Therefore (see [15]) }\left(P^{\varepsilon} \vec{u}_{\varepsilon}\right)_{\varepsilon}
$$

converges to $\overrightarrow{0}$ in the appropriate space $L D(\Omega)$, hence in $\left(L^{1}(\Omega)\right)^{N}$ and finally in $\left(H_{0}^{1}(\Omega)\right)^{N}$. The preceding equality implies that $\left(g(\varepsilon) P^{\varepsilon} \vec{u}_{\varepsilon}\right)_{\varepsilon}$ converges to $\overrightarrow{0}$ in $L D(\Omega)$ and in $\left(L^{\mathbf{1}}(\Omega)\right)^{N}$.

Multiply the preceding equality by $g(\varepsilon)$. One now proves that $\left((g(\varepsilon))^{2} P^{\varepsilon} \vec{u}_{\varepsilon}\right)_{\varepsilon}$ converges to $\overrightarrow{0}$ in $\left(L^{1}(\Omega)\right)^{N}$ and in $\left(H_{0}^{1}(\Omega)\right)^{N}$. The assertion is proved in a recurrent way.

\section{The case $g>0$ and $r_{\varepsilon}=a \varepsilon(0<a<1 / 2)$ : \\ The nonlinear Darcy's law.}

Notice first that in the present case, the coefficient $g$ has to be changed into $g \varepsilon$ [10]. Let us denote $\vec{u}_{\varepsilon}$ the solution of the minimization problem

$$
\begin{aligned}
& \operatorname{Min}_{\left(H_{0}^{1}(\Omega)\right)^{N}}\left(\frac { 1 } { 2 } \int _ { \Omega } \sum _ { i j } \left(e_{i j}(\vec{u})^{2} d x+g \varepsilon \int_{\Omega}\left(\sum_{i j}\left(e_{i j}(\vec{u})\right)^{2}\right)^{1 / 2} d x+\delta_{V\left(\Omega_{\varepsilon}\right)}(\vec{u})\right.\right. \\
& \left.-\int_{\Omega} \vec{f} \cdot \vec{u} d x\right)
\end{aligned}
$$

According to the results obtained in Theorem 2.2, $\left(P^{\varepsilon} \vec{u}_{\varepsilon}\right)_{\varepsilon}$ converges to $\overrightarrow{0}$ in the weak topology (and in fact the strong topology) of $\left(H_{0}^{1}(\Omega)\right)^{N}$, since 


\section{A. Brillard}

$\alpha_{N}$ is equal to $+\infty$, in this case. The purpose of this paragraph is to study . the rate of convergence.

LeMмA 3.1. - The sequence $\left(\varepsilon^{-2} P^{\varepsilon} \vec{u}_{\varepsilon}\right)_{\varepsilon}$ is bounded in $\left(L^{2}(\Omega)\right)^{N}$. $\varepsilon^{-2} P^{\varepsilon} \vec{u}_{\varepsilon}$ is the solution of the minimization problem

$$
\underset{\vec{v} \in\left(L^{2}(\Omega)\right)^{N}}{\operatorname{Min}}\left(G^{\varepsilon}(\vec{v})-\int_{\Omega} \vec{f} \vec{v} d x\right),
$$

where $G^{\varepsilon}$ is the functional defined on $\left(L^{2}(\Omega)\right)^{N}$ by

$$
G^{\varepsilon}(\vec{v})=\frac{\varepsilon^{2}}{2} \int_{\Omega} \sum_{i j}\left(e_{i j}(\vec{v})\right)^{2} d x+\varepsilon g \int_{\Omega}\left(\sum_{i j}\left(e_{i j}(\vec{v})\right)^{2}\right)^{1 / 2} d x+\delta_{V\left(\Omega_{\varepsilon}\right)}(\vec{v}) .
$$

\section{Proof of Lemma 3.1}

The first assertion follows from (13), thanks to Korn's inequality in $\Omega$, the positivity of $g$ and the following estimate of Poincaré's inequality in $\Omega_{\varepsilon}$, the proof of which is trivial :

LEMMA 3.2. - There exists a strictly positive constant $C$ such that :

$\forall u \in H^{1}(Y), u=0$ on $a T: \int_{Y \backslash \overline{a T}} u^{2} d y \leq C \int_{Y \backslash \overline{a T}}|\operatorname{grad} u|^{2} d y$,

$\forall u \in H^{1}\left(Y_{\varepsilon}\right), u=0$ on $T_{\varepsilon}: \int_{Y_{\varepsilon} \backslash \bar{T}_{\varepsilon}} u^{2} d x \leq C \varepsilon^{2} \int_{Y_{\varepsilon} \backslash \bar{T}_{\varepsilon}}|\operatorname{grad} u|^{2} d x$, $\forall u \in H_{0}^{1}\left(\Omega_{\varepsilon}\right): \int_{\Omega_{c}} u^{2} d x \leq C \varepsilon^{2} \int_{\Omega_{\varepsilon}}|\operatorname{grad} u|^{2} d x$.

In order to describe the limit of the sequence $\left(\varepsilon^{-2} P^{\varepsilon} \vec{u}_{\varepsilon}\right)_{\varepsilon}$, in the weak topology of $\left(L^{2}(\Omega)\right)^{N}$, one has to compute the epi-limit $G$ of the sequence $\left(G^{\varepsilon}\right)_{\varepsilon}$ in this topology. Notice that the functional $: \vec{v} \longrightarrow \int_{\Omega} \vec{f} \cdot \vec{v} d x$ is continuous for the weak topology of $\left(L^{2}(\Omega)\right)^{N}$ (see Theorem 1.6 and Proposition 1.7).

From the divergence-free condition contained in $G^{\varepsilon}$, one first deduces that the domain of $G$ in contained in $H(\Omega)$, the closure of $V(\Omega)$, with respect to the (weak) topology of $\left(L^{2}(\Omega)\right)^{N}$. Hence, one has to prove the following assertions, deduced from (7) and (8) :

for every $\vec{v}$ in $H(\Omega)$, there exists a sequence $\left(P^{\varepsilon} \vec{v}_{\varepsilon, 0}\right)_{\varepsilon}$ converging to $\vec{v}$ in the weak topology of $\left(L^{2}(\Omega)\right)^{N}$, with $\vec{v}_{\varepsilon, 0}$ in $V\left(\Omega_{\varepsilon}\right)$, such that

$$
\underset{\varepsilon \rightarrow 0}{\limsup } \quad G^{\varepsilon}\left(\vec{v}_{\varepsilon, 0}\right) \leq G(\vec{v}),
$$


for every $\vec{v}$ in $H(\Omega)$ and for every sequence $\left(P^{\varepsilon} \vec{v}_{\varepsilon}\right)_{\varepsilon}$ converging to $\vec{v}$ in the weak topology of $\left(L^{2}(\Omega)\right)^{N}$, with $\vec{v}_{\varepsilon}$ in $V\left(\Omega_{\varepsilon}\right)$ :

$$
\liminf _{\varepsilon \rightarrow 0} G^{\varepsilon}\left(\vec{v}_{\varepsilon}\right) \geq G(\vec{v}) .
$$

The verification of (15) and (16) requires the local character of $G^{\varepsilon}$ and the density of the set of piecewise constant functions in $\left(L^{2}(\Omega)\right)^{N}$ :

Definition 3.3. - A function $\vec{u}$ in $\left(L^{2}(\Omega)\right)^{N}$ is called piecewise constant if there exists a finite family $\left(\Omega_{p}\right)_{1 \leq p \leq p(\vec{u})}$ of smooth open subsets of $\Omega$, with $\Omega \backslash \overline{U_{p}}$ negligible, such that $\vec{u}$ is a constant $\vec{u}_{p}$ on $\Omega_{p}$.

The verification of (15) and (16) also requires the study of the local minimization problem :

For every $\xi$ in $R^{N}$, let $\vec{z}_{\xi}$ be the solution of

$\operatorname{Min}$

$$
\begin{aligned}
& \left(\frac { 1 } { 2 } \int _ { Y \backslash \overline { a T } } \sum _ { i j } \left(e_{i j}(\vec{z})^{2} d y+\right.\right. \\
& \left.+g \int_{Y \backslash \overline{a T}}\left(\sum_{i j}\left(e_{i j}(\vec{z})\right)^{2}\right)^{1 / 2} d y-\xi \cdot \widetilde{\vec{z}}\right) .
\end{aligned}
$$

$\vec{z} Y$-periodic

$\operatorname{div} \vec{z}=0$ on $Y$

$\vec{z}=\overrightarrow{0}$ on $a T$

Let $A_{0}$ be the function : $\left(\begin{array}{ccc}R^{N} & \longrightarrow & R^{N} \\ \xi & \longrightarrow & \widetilde{\vec{z}}_{\xi}\end{array}\right)$

The properties of $A_{0}$ are summarized in the following Lemma :

Lemma 3.4. - (see [10] and Appendix 2)

a) For every $\lambda$ in $R^{N}$, there exists $\xi(\lambda)$ in $R^{N}$ such that $: A_{0}(\xi(\lambda))=\lambda$.

b) this $\xi(\lambda)$ is not unique, but if $\xi_{1}$ and $\xi_{2}$ belong to $A_{0}^{-1}(\lambda)$ :

$$
\begin{aligned}
& \frac{1}{2} \int_{Y \backslash \overline{a T}} \sum_{i j}\left(e_{i j}\left(\vec{z}_{\xi_{1}}\right)\right)^{2} d y+g \int_{Y \backslash \overline{a T}}\left(\sum_{i j}\left(e_{i j}\left(\vec{z}_{\xi_{1}}\right)\right)^{2}\right)^{1 / 2} d y= \\
& \quad=\frac{1}{2} \int_{Y \backslash \overline{a T}} \sum_{i j}\left(e_{i j}\left(\vec{z}_{\xi_{2}}\right)\right)^{2} d y+g \int_{Y \backslash \overline{a T}}\left(\sum_{i j}\left(e_{i j}\left(\vec{z}_{\xi_{2}}\right)\right)^{2}\right)^{1 / 2} d y,
\end{aligned}
$$

c) there exist two constants $c, C$ independant of $\xi$ such that :

$$
\begin{gathered}
c|\xi|^{2} \leq A_{0} \xi \cdot \xi \leq C|\xi|^{2}, \quad(0<c \leq C) \\
-53-
\end{gathered}
$$




\section{A. Brillard}

d) there exist a symmetric matrix valued function $m_{\xi}$ and a function $q_{\xi}$ verifying

$\sum_{i}\left(m_{\xi}\right)_{i i}=0$.

$\sum_{i j}\left(m_{\xi}\right)_{i j}\left(m_{\xi}\right)_{i j} \leq 1$ a.e. in $Y$,

$\sum_{i j}\left(m_{\xi}\right)_{i j} e_{i j}\left(\vec{z}_{\xi}\right)=\left(\sum_{i j}\left(e_{i j}\left(\vec{z}_{\xi}\right)\right)^{2}\right)^{1 / 2}$ a.e. in $Y$,

$-\sum_{j} D_{j} e_{i j}\left(\vec{z}_{\xi}\right)-g \sum_{j} D_{j}\left(m_{\xi}\right)_{i j}=-D_{i} q_{\xi}+\xi_{i}$ in $Y$.

Let us denote by $j_{0}$ the function from $R^{N}$ into $R$ defined by

$$
\begin{aligned}
j_{0}(\eta) & =\frac{1}{2} \int_{Y \backslash \overline{a T}} \sum_{i j}\left(e_{i j}\left(\vec{z} A_{0}^{-1} \eta\right)\right)^{2} d y+ \\
& +g \int_{Y \backslash \overline{a T}}\left(\sum_{i j}\left(e_{i j}\left(\vec{z} A_{0}^{-1} \eta\right)\right)^{2}\right)^{1 / 2} d y .
\end{aligned}
$$

(this is justified by Lemma $3.4 \mathrm{~b}$ )).

From (17) and Lemma $3.4 \mathrm{c}$ ), one immediately infers that $j_{0}$ has a quadratic growth, namely : $j_{0}(\eta) \leq C+C^{\prime}|\eta|^{2}\left(0 \leq C, C^{\prime}\right)$. Moreover, an elementary computation (deduced from (17)) proves that $j_{0}$ is convex.

Consequently, the functional $\int_{\Omega} j_{0}(\vec{v}(x)) d x$ is well-defined on $\left(L^{2}(\Omega)\right)^{N}$. Moreover, this functional $G$ is convex and continuous, with respect to the strong topology of $\left(L^{2}(\Omega)\right)^{N}$.

Verification of (15) with $G(\vec{v})=\int_{\Omega} j_{0}(\vec{v}(x)) d x$.

Take any $\vec{v}$ in $H(\Omega)$. Let $\left(\vec{v}_{n}\right)_{n}$ be any sequence of piecewise constant functions converging to $\vec{v}$ in the strong topology of $\left(L^{2}(\Omega)\right)^{N}$ :

$\vec{v}_{n}=\vec{v}_{n p}$ on $\Omega_{n p}(1 \leq p \leq p(n))$, with $\Omega \backslash \bar{\cup}_{p} \Omega_{n p}$ negligible. For every $p$ Lemma 3.4 implies the existence of an $Y$-periodic function $\vec{z}_{\xi\left(\vec{v}_{n p}\right)}$ such that the mean value $\widetilde{\vec{z}}_{\xi\left(\vec{v}_{n p}\right)}$ is equal to $\vec{v}_{n p}$. Then we define $\vec{z}_{\xi\left(\vec{v}_{n p}\right)}$ as the $Y_{\varepsilon}$-periodic function equal to $\vec{z}_{\xi\left(\vec{v}_{n p}\right)}(\dot{\bar{\varepsilon}})$ in $Y_{\varepsilon}$ and $\varepsilon$-periodically extented in $\Omega_{n p}$. 
Lemma 4.1 of [12] implies that $\left(\vec{z}_{\xi\left(\vec{v}_{n p}\right)}\right)_{\epsilon}$ converges in the weak topology of $\left(L^{2}\left(\Omega_{n p}\right)\right)^{N}$ to $\widetilde{\vec{z}}_{\xi\left(\vec{v}_{n p}\right)}=\vec{v}_{n p}$.

The main difficulty of the problem is to gather all these different $Y_{\varepsilon}$-periodic functions $\vec{z}_{\xi\left(\vec{v}_{n p}\right)}^{\varepsilon}$. This may be done in the following way.

First, define $\sum_{\varepsilon}$ as the set $\left\{x \in \Omega / d\left(x, \partial \Omega \cup \underset{p}{\cup} \partial \Omega_{n p}\right)<\varepsilon\right\}$.

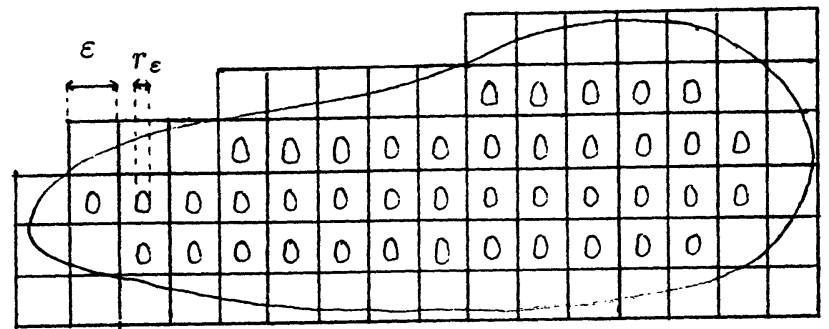

Figure2

Let $\vec{z}_{n \varepsilon}$ be the solution of the Stokes problem in $\sum_{\varepsilon}$, with boundary conditions equal either to $\overrightarrow{0}$ (on $\partial \Omega$ or on the boundary of the pieces of inclusions included in $\sum_{\varepsilon}$ ) or $\vec{z}_{\xi\left(v_{n p}\right)}^{\in}$ (on $\left.\partial \Omega_{n p} \cap \partial \sum_{\varepsilon}\right)$.

One verifies that the sequence $\left(\varepsilon\left\|\vec{z}_{n \varepsilon}\right\|_{\left.\left.H^{1}\left(\Sigma_{\varepsilon}\right)\right)^{N}\right)_{\varepsilon}}\right.$ converges to 0 , when $\varepsilon$ converges to 0 . Then, one deduces, as in Lemma 3.2, that the sequence $\left(\left\|\vec{z}_{n \varepsilon}\right\|_{\left.\left(L^{2}\left(\Sigma_{\varepsilon}\right)\right)^{N}\right)_{\varepsilon}}\right.$ converges to 0 .

Finally, we define $: \vec{v}_{n, \varepsilon, 0}=\mid \begin{array}{ll}\vec{z}_{\xi\left(\vec{v}_{n p}\right)}^{*} & \text { in } \Omega_{n p} \backslash \overline{\Sigma_{\varepsilon}}, \\ \vec{z}_{n \varepsilon} & \text { in } \Sigma_{\varepsilon} .\end{array}$

Trivially $\vec{v}_{n, \varepsilon, 0}$ belongs to $V\left(\Omega_{\varepsilon}\right)$ and $\left(P^{\varepsilon} \vec{v}_{n, \varepsilon, 0}\right)_{\varepsilon}$ converges to $\vec{v}_{n}$ in the weak topology of $\left(L^{2}(\Omega)\right)^{N}$. The computation of $G^{\varepsilon}\left(\vec{v}_{n, \varepsilon, 0}\right)$ is quite trivial, since

$$
\begin{aligned}
G^{\varepsilon}\left(\vec{v}_{n, \varepsilon, 0}\right) & \leq \sum_{p} \frac{\varepsilon^{2}}{2} \int_{\Omega_{n p}} \sum_{i j}\left(e_{i j}\left(\vec{z}_{\xi\left(\vec{v}_{n p}\right)}^{*}\right)\right)^{2} d x \\
& +\varepsilon g \int_{\Omega_{n p}}\left(\sum_{i j}\left(e_{i j}\left(\vec{z}_{\xi\left(\vec{v}_{n p}\right)}^{*}\right)\right)^{2}\right)^{1 / 2} d x \\
& +\frac{\varepsilon^{2}}{2} \int_{\Sigma_{\varepsilon}} \sum_{i j}\left(e_{i j}\left(\vec{z}_{n \varepsilon}\right)\right)^{2} d x+\varepsilon g \int_{\Sigma_{\varepsilon}}\left(\sum_{i j}\left(e_{i j}\left(\vec{z}_{n \varepsilon}\right)\right)^{2}\right)^{1 / 2} d x
\end{aligned}
$$




\section{A. Brillard}

Hence, from the definition of $\vec{z}_{\xi\left(\vec{v}_{n p}\right)}$ and (18):

$$
\underset{\varepsilon \rightarrow 0}{\limsup } G^{\varepsilon}\left(\vec{v}_{n, \varepsilon, 0}\right) \leq \sum_{p} \int_{\Omega_{n p}} j_{0}\left(\vec{v}_{n p}\right) d x=\int_{\Omega} j_{0}\left(\vec{v}_{n}(x)\right) d x=G\left(\vec{v}_{n}\right) .
$$

Therefore :

$$
\limsup _{n \rightarrow+\infty} \limsup _{\varepsilon \rightarrow 0} G^{\varepsilon}\left(\vec{v}_{n, \varepsilon, 0}\right) \leq G(\vec{v}) .
$$

The diagonalization argument of Corollary 1.16 [1] implies the existence of a subsequence $(n(\varepsilon))_{\varepsilon}$ growing to $+\infty$ such that $\left(P^{\varepsilon} \vec{v}_{n(\varepsilon), \varepsilon, 0}\right)_{\varepsilon}$ converges to $\vec{v}$, in the weak topology of $\left(L^{2}(\Omega)\right)^{N}$, and

$$
\limsup _{\varepsilon \rightarrow 0} G^{\varepsilon}\left(\vec{v}_{n(\varepsilon), \varepsilon, 0}\right) \leq G(\vec{v})
$$

(15) is proved, taking $\vec{v}_{\varepsilon, 0}=\vec{v}_{n(\varepsilon), \varepsilon, 0}$.

Verification of (16) with : $G(\vec{v})=\int_{\Omega} j_{0}(\vec{v}(x)) d x$.

Choose any $\vec{v}$ in $H(\Omega)$. Let $\left(\vec{v}_{n}\right)_{n}$ be any sequence of piecewise constant functions in $\Omega$, converging to $\vec{v}$ in the strong topology of $\left(L^{2}(\Omega)\right)^{N}: \vec{v}_{n}=\vec{v}_{n p}$ in $\Omega_{n p}$ with $\Omega \backslash \overline{\bigcup_{p} \Omega_{n p}}$ negligible.

Suppose that $\left(P^{\varepsilon} \vec{v}_{\varepsilon}\right)_{\varepsilon}$ converges to $\vec{v}$ in the weak topology of $\left(L^{2}(\Omega)\right)^{N}$, with $\vec{v}_{\varepsilon}$ in $V\left(\Omega_{\varepsilon}\right)$. We may suppose that :

$$
\limsup _{\varepsilon \rightarrow 0} G^{\varepsilon}\left(\vec{v}_{\varepsilon}\right)<+\infty
$$

otherwise, (16) is trivially true.

For every $p$, we choose a smooth function $\emptyset_{n p}$ in $C_{0}^{\infty}\left(\Omega_{n p}\right)$ satisfying $0 \leq \emptyset_{n p} \leq 1$, and we write :

$$
\begin{gathered}
G^{\varepsilon}\left(\vec{v}_{\varepsilon}\right) \geq \sum_{p}\left\{\frac{\varepsilon^{2}}{2} \int_{\Omega_{n p}} \sum_{i j}\left(e_{i j}\left(\vec{v}_{\varepsilon}\right)\right)^{2} \emptyset_{n p} d x\right. \\
\left.\quad+\varepsilon g \int_{\Omega_{n p}}\left(\sum_{i j}\left(e_{i j}\left(\vec{v}_{\varepsilon}\right)\right)^{2}\right)^{1 / 2} \emptyset_{n p} d x\right\} \\
\geq \sum_{p}\left\{\frac{\varepsilon^{2}}{2} \int_{\Omega_{n p}} \sum_{i j}\left(e_{i j}\left(\vec{z}_{\xi\left(\vec{v}_{n p}\right)}^{\varepsilon}\right)\right)^{2} \emptyset_{n p} d x\right. \\
-56-
\end{gathered}
$$


Asymptotic behaviour of a viscoplastic bingham fluid in porous media

$$
\begin{aligned}
& +\varepsilon g \int_{\Omega_{n p}}\left(\sum_{i j}\left(e_{i j}\left(\vec{z}_{\xi\left(\vec{v}_{n p}\right)}^{\epsilon}\right)\right)^{2}\right)^{1 / 2} \emptyset_{n p} d x \\
& +\varepsilon^{2} \int_{\Omega_{n p}} \sum_{i j} e_{i j}\left(\vec{z}_{\xi\left(\vec{v}_{n p}\right)}^{\epsilon}\right) e_{i j}\left(\vec{v}_{\varepsilon}-\vec{z}_{\xi\left(\vec{v}_{n p}\right)}^{\epsilon}\right) \emptyset_{n p} d x \\
& +\varepsilon g \int_{\Omega_{n p}}\left(\sum_{i j}\left(e_{i j}\left(\vec{v}_{\varepsilon}\right)\right)^{2}\right)^{1 / 2} \emptyset_{n p} d x \\
& \left.-\varepsilon g \int_{\Omega_{n p}}\left(\sum_{i j}\left(e_{i j}\left(\vec{z}_{\xi\left(\vec{v}_{n p}\right)}^{\in}\right)\right)^{2}\right)^{1 / 2} \emptyset_{n p} d x\right\} .
\end{aligned}
$$

From the definition of $\vec{z}_{\xi\left(\vec{v}_{n p}\right)}^{\varepsilon}$, the smoothness of $\emptyset_{n p}$ and the definition of $j_{0}(18)$, we derive :

$$
\begin{aligned}
\lim _{\varepsilon \rightarrow 0}\left(\sum_{p} \frac{\varepsilon^{2}}{2} \int_{\Omega_{n p}}\right. & \sum_{i j}\left(e_{i j}\left(\vec{z}_{\xi\left(\vec{v}_{n p}\right)}^{e}\right)\right)^{2} \emptyset_{n p} d x \\
& \left.+\varepsilon g \int_{\Omega_{n p}}\left(\sum_{i j}\left(e_{i j}\left(\vec{z}_{\xi\left(\vec{v}_{n p}\right)}^{\epsilon}\right)\right)^{2}\right)^{1 / 2} \emptyset_{n p} d x\right) \\
& =\sum_{p} \int_{\Omega_{n p}} j_{0}\left(\vec{v}_{n p}\right) \emptyset_{n p} d x .
\end{aligned}
$$

From Lemma 3.4.d) and the smoothness of $\emptyset_{n p}$, we derive :

$$
\begin{aligned}
& \liminf _{\varepsilon \rightarrow 0} \sum_{p}\left\{\varepsilon^{2} \int_{\Omega_{n p}} \sum_{i j} e_{i j}\left(\vec{z}_{\xi\left(\vec{v}_{n p}\right)}^{*}\right) e_{i j}\left(\vec{v}_{\varepsilon}-\vec{z}_{\xi\left(\vec{v}_{n p}\right)}^{\in}\right) \emptyset_{n p} d x\right. \\
& +\varepsilon g \int_{\Omega_{n p}}\left(\sum_{i j}\left(e_{i j}\left(\vec{v}_{\varepsilon}\right)\right)^{2}\right)^{1 / 2} \emptyset_{n p} d x \\
& \left.\quad-\varepsilon g \int_{\Omega_{n p}}\left(\sum_{i j}\left(e_{i j}\left(\vec{z}_{\xi\left(v_{n p}\right)}^{e}\right)\right)^{2}\right)^{1 / 2} \emptyset_{n p} d x\right\} \\
& \geq \sum_{p} \int_{\Omega_{n p}} \xi\left(\vec{v}_{n p}\right)\left(\vec{v}-\vec{v}_{n p}\right) \emptyset_{n p} d x .
\end{aligned}
$$

Let $\emptyset_{n p}$ increase to the characteristic function $\mathcal{X}_{\Omega_{n p}}$ of $\Omega_{n p}$ :

$$
\begin{gathered}
\liminf _{\varepsilon \rightarrow 0} G^{\varepsilon}\left(\vec{v}_{\varepsilon}\right) \geq \sum_{p}\left\{\int_{\Omega_{n p}} j_{0}\left(\vec{v}_{n p}\right) d x+\int_{\Omega_{n p}} \xi\left(\vec{v}_{n p}\right)\left(\vec{v}-\vec{v}_{n p}\right) d x\right\} . \\
\geq \int_{\Omega} j_{0}\left(\vec{v}_{n}\right) d x+\int_{\Omega} \xi\left(\vec{v}_{n}\right)\left(\vec{v}-\vec{v}_{n}\right) d x . \\
-57-
\end{gathered}
$$




\section{A. Brillard}

Let $n$ increase to $+\infty:(16)$ is proved, thanks to the continuity of $G$, with respect to the strong topology of $\left(L^{2}(\Omega)\right)^{N}$.

Finally, we have proved :

THEOREM 3.5. - The sequence $\left(G^{\varepsilon}\right)_{\varepsilon}$ of functionals defined by (14) epiconverges in the weak topology of $\left(L^{2}(\Omega)\right)^{N}$ to the functional $G$ defined on $\left(L^{2}(\Omega)\right)^{N}$ by :

$$
G(\vec{v})=\int_{\Omega} j_{0}(\vec{v}(x)) d x+\delta_{H(\Omega)}(\vec{v}),
$$

where $j_{0}$ is defined by (18).

Through Theorem 1.6, we deduce of Theorem 3.5 :

CoROLlary 3.6. - There exists a function $p_{1}$ in $L^{2}(\Omega) / R$ such that the sequence $\left(\frac{1}{\varepsilon^{2}} P^{\varepsilon} \vec{u}_{\varepsilon}\right)_{\varepsilon}$ converges in the weak topology of $\left(L^{2}(\Omega)\right)^{N}$ to $A_{0}\left(\vec{f}-\operatorname{grad} p_{1}\right)$

Moreover,

$$
\left(\frac{1}{\varepsilon^{2}} \int_{\Omega} \sum_{i j}\left(e_{i j}\left(P^{\varepsilon} \vec{u}_{\varepsilon}\right)\right)^{2} d x+\frac{g}{\varepsilon} \int_{\Omega}\left(\sum_{i j}\left(e_{i j}\left(P^{\varepsilon} \vec{u}_{\varepsilon}\right)\right)^{2}\right)^{1 / 2} d x\right)_{\varepsilon}
$$

converges to

$$
\begin{gathered}
\int_{\Omega}\left(\frac{1}{2} \int_{Y \backslash \overline{a T}} \sum_{i j}\left(e_{i j}\left(\vec{z}_{\vec{f}-\operatorname{grad} p_{1}}\right)\right)^{2} d y+\right. \\
\left.+g \int_{Y \backslash \overline{a T}}\left(\sum_{i j}\left(e_{i j}\left(\vec{z}_{\vec{f}-\operatorname{grad} p_{1}}\right)\right)^{2}\right)^{1 / 2} d y\right) d x .
\end{gathered}
$$

Remark 3.7.- J.L. Lions and E. SANChEz-FALEncia proved in [10] that $p_{1}$ is not unique in $L^{2}(\Omega) / R$. In fact, if $\xi$ is sufficiently small, $A_{0} \xi$ is equal to 0 . (see [10] 4.3). However, two determinations of the limit pressure $p_{1}$ provide the same mean velocity (see Appendix 2).

Remark 3.8. - In the case $N=2$, the construction of the test function $\vec{v}_{\varepsilon, 0}$ satisfying (15) may be done in the following way. First, one deduces from Lemma 2.5 of [14], through a trivial change of scale, the following property of $Y_{\varepsilon}$-periodic, divergence-free functions. 
Asymptotic behaviour of a viscoplastic bingham fluid in porous media

LEMma 3.9. - For every $Y_{\varepsilon}$-periodic, divergence-free function $\widehat{z}$ in $\left(L^{2}\left(Y_{\varepsilon}^{2}\right)\right)$, there exists an $Y_{\varepsilon}-$ periodic function $\widehat{z}$ in $H^{1}\left(Y_{\varepsilon}\right)$ verifying :

$\operatorname{curl} \widehat{z}=\left(D_{2} \widehat{z},-D_{1} \widehat{z}\right)=\vec{z}$ in $Y_{\varepsilon}$,

$\|\widehat{z}\|_{L^{2}\left(Y_{e}\right)} \leq C \varepsilon\|\vec{z}\|_{\left(L^{2}\left(Y_{e}\right)\right)^{2}} ;\|\operatorname{grad} \hat{z}\|_{\left(L^{2}\left(Y_{e}\right)\right)^{2}} \leq C\|\vec{z}\|_{\left(L^{2}\left(Y_{e}\right)\right)^{2}}$,

where $C$ is a constant independant of $\varepsilon$ and $\vec{z}$.

We also need the following restriction operator $R^{\varepsilon}$ :

Proposition 3.10 [13]. - There exists a linear restriction operator $R^{\varepsilon}$ from $\left(H_{0}^{1}(\Omega)\right)^{N}$ into $\left(H_{0}^{1}\left(\Omega_{\varepsilon}\right)\right)^{N}$ satisfying :

a) for every $\vec{u}$ in $\left(H_{0}^{1}\left(\Omega_{\varepsilon}\right)\right)^{N}: R^{\varepsilon} P^{\varepsilon} \vec{u}=\vec{u}$ ( $P^{\varepsilon}$ is the canonical extension operator),

b) $R^{\varepsilon}(V(\Omega)) \subset V\left(\Omega_{\varepsilon}\right)$,

b) there exists a constant $C$ independant of $\varepsilon$ and $\vec{u}$ in $\left(H_{0}^{1}(\Omega)\right)^{N}$ such that

$\left\|R^{\varepsilon} \vec{u}\right\|_{\left(L^{2}\left(\Omega_{\varepsilon}\right)\right)^{N}} \leq C\|\vec{u}\|_{\left(L^{2}(\Omega)\right)^{N}+C \varepsilon\|\operatorname{grad} \vec{u}\|_{\left(L^{2}(\Omega)\right)^{N^{2}}}}$

$\left\|\operatorname{grad} R^{\varepsilon} \vec{u}\right\|_{\left(L^{2}\left(\Omega_{\varepsilon}\right)\right)^{N^{2}}} \leq \frac{C}{\varepsilon}\|\vec{u}\|_{\left(L^{2}(\Omega)\right)^{N}}+C\|\operatorname{grad} \vec{u}\|_{\left(L^{2}(\Omega)\right)^{N^{2}}}$

And then we define :

$$
\vec{v}_{\varepsilon, 0}=R^{\varepsilon}\left(\operatorname{curl}\left(\sum_{p} \widehat{z}_{\xi\left(\vec{v}_{\left.n_{p}\right)}\right)}^{\varepsilon} \chi_{\Omega_{n p}} \emptyset_{n \varepsilon}\right)\right),
$$

where $\widetilde{z}_{\xi\left(\vec{v}_{n p}\right)}^{\epsilon}$ is the function associated to the $Y_{\varepsilon}$-periodic, divergence-free function $\widehat{z}_{\xi\left(v_{n p}\right)}^{\varepsilon}$, by Lemma 3.9 and $\emptyset_{n \varepsilon}$ is a smooth function in $C_{0}^{1}(\bar{\Omega})$ and identically equal to 1 in $\Omega \backslash \bar{\Sigma}_{\varepsilon}$.

IV - Convergence of the internal pressure when

$$
r_{\varepsilon}=a \varepsilon(0<a<1 / 2)
$$

THEOREM 4.1. - There exists an extension $\bar{p}_{\varepsilon}$ of the internal pressure of the Bingham fluid, such that $\left(\bar{p}_{\varepsilon}\right)_{\varepsilon}$ converges, in the strong topology of $L^{2}(\Omega) / R$. 


\section{A. Brillard}

Proof of Theorem 4.1.

Through Proposition 3.10b), define (as in [13]) $\operatorname{grad} \bar{p}_{\varepsilon}$ the element of $\left(H^{-1}(\Omega)\right)^{N}$ satisfying :

$$
<\operatorname{grad} \bar{p}_{\varepsilon}, \vec{u}>=<\operatorname{grad} p_{\varepsilon}, R^{\varepsilon} \vec{u}>
$$

for every $\vec{u}$ in $\left(H_{0}^{1}(\Omega)\right)^{N}$.

From (13), one deduces the following estimate :

$$
\left\|\operatorname{grad} p_{\varepsilon}\right\|_{\left.H^{-1}\left(\Omega_{c}\right)\right)^{N}} \leq C \varepsilon .
$$

Then Proposition 3.10c) implies the boundedness of $\left(\operatorname{grad} \bar{p}_{\varepsilon}\right)_{\varepsilon}$ in $\left(H^{-1}(\Omega)\right)^{N}$.

As in [13], we then prove the convergence of $\left(\bar{p}_{\varepsilon}\right)_{\varepsilon}$, in the strong topology of the quotient space $L^{2}(\Omega) / R$, to some limit point $p_{2}$. We conjecture that this limit point $p_{2}$ is, in fact, one "limit pressure" appearing in the limit law established in Corollary 3.6 (see Remark 3.7).

\section{APPENDIX 1 : The proof of Proposition 2.3.}

Let us first mention the following property of the divergence-free functions (which is an immediate consequence of Theorem 4.9 of [11], see also [14]) :

Proposition A.1. - a) Suppose $N=3:$ for every divergence-free function $\vec{w}$ in $\left(L^{2}(B(r))\right)^{3}$, there exists a divergence-free function $\widehat{\vec{w}}$ in $\left(H^{1}(B(r))\right)^{3}$ satisfying

$$
\begin{aligned}
& \widehat{\vec{w}} \cdot \vec{n}=0 \quad \text { on } \partial B(r)(\vec{n} \text { is the outer normal to } \partial B(r)), \\
& \text { curl } \widehat{\vec{w}}=\vec{w} \text { in } B(r), \\
& \|\widehat{\vec{w}}\|_{\left(L^{2}(B(r))\right)^{3}} \leq C r\|\vec{w}\|_{\left(L^{2}(B(r))\right)^{3},} \\
& \|\operatorname{grad} \widehat{\vec{w}}\|_{\left(L^{2}(B(r))\right)^{9}} \leq C\|\vec{w}\|_{\left(L^{2}(B(r))\right)^{3}},
\end{aligned}
$$

b) Suppose $N=2$ : for every divergence-free function $\vec{w}$ in $\left(L^{2}(B(r))\right)^{2}$, there exists a function $\widehat{w}$ in $H^{1}(B(r))$ satisfying :

$$
\begin{aligned}
& \widehat{w}=0 \quad \text { on } \partial B(r), \\
& \text { curl } \widehat{w}=w \quad \text { in } B(r), \\
& \|\widehat{w}\|_{L^{2}(B(r))} \leq C r\|\vec{w}\|_{\left(L^{2}(B(r))\right)^{2}}, \\
& \|\operatorname{grad} \widehat{w}\|_{\left(L^{2}(B(r))\right)^{2}} \leq C\|\vec{w}\|_{\left(L^{2}(B(r))\right)^{2}},
\end{aligned}
$$


Asymptotic behaviour of a viscoplastic bingham fluid in porous media

where $C$ is a constant independant of $r(r>0)$ and $\vec{w}$.

Let us now prove the Proposition 2.3. Suppose that $T$ is equal to $B(1)$ and define

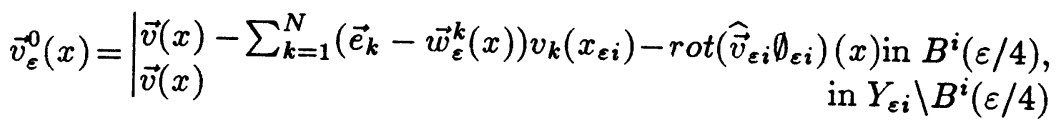

where $x_{\varepsilon i}$ is the center of $Y_{\varepsilon i}$ and $B^{i}(\varepsilon / 4)$ (the $i-$ the cell and the $i-$ th ball), $\widehat{\vec{v}}_{\varepsilon i}$ is the divergence-free function associated to $\vec{v}(\cdot)-\vec{v}\left(x_{\varepsilon i}\right)$ in $B^{i}\left(r_{\varepsilon}^{2 / 3}\right)$, by Proposition A.1, $\emptyset_{\varepsilon i}$ is a cut-off function in $C^{\infty}(\Omega ;[0,1])$ whose support is contained in $B^{i}\left(r_{\varepsilon}^{2 / 3}\right)$ and identically equal to 1 in $B^{i}\left(r_{\varepsilon}\right)$.

In the present case, the solution $\left(\vec{w}_{\varepsilon}^{k}, q_{\varepsilon}^{k}\right)$ of the minimization problem (4) is computable in terms of radial functions (see [5]). The assertions of Proposition 2.3 are immediate consequences of these computations.

When $T$ is a smooth open subset of the unit ball $B(1)$ of $R^{N}$, one has to modify the test functions $\vec{v}_{\varepsilon}^{0}$, given in (19), in order to use the following pointwise estimates on the solution $\left(\vec{w}_{\varepsilon}^{k}, q_{\varepsilon}^{k}\right)$ of $(4)$ :

Proposition A.2 ([11] Lemma 2.2). - for every $s_{\varepsilon}\left(s_{\varepsilon}<\varepsilon\right)$ and $c$ $(c>0)$, there exists a constant $C$ such that if $N=3$ : for every $x$ in $B\left(s_{\varepsilon}\right)$ verifying $d\left(x, T_{\varepsilon}\right)>c r_{\varepsilon}$

$$
\begin{gathered}
\left|\vec{e}_{k}-\vec{w}_{\varepsilon}^{k}(x)\right| \leq C \frac{r_{\varepsilon}}{d\left(x, T_{\varepsilon}\right)} ;\left|D_{i} \vec{w}_{\varepsilon}^{k}(x)\right| \leq C \frac{r_{\varepsilon}}{d\left(x, T_{\varepsilon}\right)^{2}} ; \\
\left|D_{i} D_{j} \vec{w}_{\varepsilon}^{k}(x)\right| \leq C \frac{r_{\varepsilon}}{d\left(x, T_{\varepsilon}\right)^{3}} ;\left|q_{\varepsilon}^{k}(x)\right| \leq C \frac{r_{\varepsilon}}{d\left(x, T_{\varepsilon}\right)^{2}}
\end{gathered}
$$

if $N=2:$ for every $x$ in $B\left(s_{\varepsilon}\right)$ verifying $d\left(x, T_{\varepsilon}\right)>\exp \left(-c\left|\log r_{\varepsilon}\right|^{1 / 2}\right)$

$$
\begin{gathered}
\left|\vec{e}_{k}-\vec{w}_{\varepsilon}^{k}(x)\right| \leq C \frac{\left|\log d\left(x, T_{\varepsilon}\right)\right|+1}{\left|\log r_{\varepsilon}\right|} ;\left|D_{i} \vec{w}_{\varepsilon}^{k}(x)\right| \leq C \frac{1}{\left|\log r_{\varepsilon}\right| d\left(x, T_{\varepsilon}\right)} ; \\
\left|D_{i} D_{j} \vec{w}_{\varepsilon}^{k}(x)\right| \leq C \frac{1}{\left|\log r_{\varepsilon}\right| d\left(x, T_{\varepsilon}\right)^{2}} ;\left|q_{\varepsilon}^{k}(x)\right| \leq C \frac{1}{\left|\log r_{\varepsilon}\right| d\left(x, T_{\varepsilon}\right)} .
\end{gathered}
$$

Define :

$$
\vec{v}_{\varepsilon}^{0}(x)=\mid \begin{aligned}
& \vec{v}(x)-\sum_{k} \operatorname{rot}\left(\widehat{\vec{w}}_{\varepsilon, i}^{k} \psi_{\varepsilon i}\right)(x) v_{k}\left(x_{\varepsilon i}\right)-\operatorname{rot}\left(\widehat{\vec{v}}_{\varepsilon i} \emptyset_{\varepsilon i}\right)(x) \operatorname{in} B^{i}(\varepsilon / 4), \\
& \vec{v}(x) \operatorname{in} Y_{\varepsilon i} \backslash B^{i}(\varepsilon / 4),
\end{aligned}
$$

where $\psi_{\varepsilon i}$ is a cut-off function whose support is contained in $B^{i}\left(\frac{\varepsilon}{4}-\frac{r_{\varepsilon}}{2}\right)$ and identically equal to 1 in $B^{i}\left(\frac{\varepsilon}{8}-\frac{r_{\varepsilon}}{2}\right)$, 
A. Brillard

$\widehat{\vec{w}}_{\varepsilon, i}^{k}$ is associated to the divergence-free function $\vec{e}_{k}-\vec{w}_{\varepsilon}^{k}$ in $B^{i}\left(\frac{\varepsilon}{4}-\frac{r_{\varepsilon}}{2}\right)$ by Proposition A.1.

The assertions of Proposition 2.3 are quite immediate consequences of these pointwise estimates.

\section{APPENDIX 2 : Proof of Lemma 3.4}

Let us first prove some properties of the function $A_{0}$ :

1) $A_{0}$ is monotone in the sense that for every $\xi_{1}$ and $\xi_{2}$ in $R^{N}$ :

$$
\left(A_{0} \xi_{1}-A_{0} \xi_{2}\right) \cdot\left(\xi_{1}-\xi_{2}\right) \geq 0
$$

From (17) we derive :

$$
\begin{aligned}
\int_{Y \backslash \overline{a T}} \sum_{i j}\left(e_{i j}\left(\vec{z}_{\xi_{1}}\right)\right) e_{i j}\left(\vec{z}_{\xi_{2}}-\vec{z}_{\xi_{1}}\right) d y & +g \int_{Y \backslash \overline{a T}}\left(\sum_{i j}\left(e_{i j}\left(\vec{z}_{\xi_{2}}\right)\right)^{2}\right)^{1 / 2} d y \\
& -g \int_{Y \backslash \overline{a T}}\left(\sum_{i j}\left(e_{i j}\left(\vec{z}_{\xi_{1}}\right)\right)^{2}\right)^{1 / 2} d y \geq \xi_{1} \cdot\left(\widetilde{\vec{z}}_{\xi_{1}}-\widetilde{\vec{z}}_{\xi_{2}}\right)
\end{aligned}
$$

and a similar inequality where $\xi_{1}$ and $\xi_{2}$ are exchanged. Add these two inequalities :

$$
\left(A_{0} \xi_{1}-A_{0} \xi_{2}\right) \cdot\left(\xi_{1}-\xi_{2}\right) \geq \int_{Y \backslash \overline{a T}} \sum_{i j}\left(e_{i j}\left(\vec{z}_{\xi_{1}}-\vec{z}_{\xi_{2}}\right)\right)^{2} d y
$$

and the monotonicity of $A_{0}$ is proved.

2) $A_{0}$ is continuous. This property of $A_{0}$ may be proved through an epi-convergence argument : let $H_{\xi}$ be the functional associated to (17).

Let $\left(\xi_{n}\right)_{n}$ be any sequence converging to $\xi$ in $R^{N}$. One trivially proves that $\left(H_{\xi_{n}}\right)_{n}$ epi-converges to $H_{\xi}$ in the strong topology of $\left(H^{1}(Y)\right)^{N}$.

Theorem 1.6 then implies that $\left(\vec{z}_{\xi_{n}}\right)_{n}$ converges to $\vec{z}_{\boldsymbol{\xi}}$ in this topology.

Hence, $\left(A_{0}\left(\xi_{n}\right)\right)_{n}$ converges to $A_{0} \xi$.

3) $\lim _{|\xi| \rightarrow+\infty} A_{0} \xi \cdot \xi /|\xi|=+\infty$.

We introduce $\vec{z}_{\xi}^{*}$ the solution of the following minimization problem : 
Asymptotic behaviour of a viscoplastic bingham fluid in porous media

Min

$$
\left(\frac{1}{2} \int_{Y \backslash \overline{a T}} \sum_{i j}\left(e_{i j}(\vec{z})\right)^{2} d y-\xi \cdot \tilde{\vec{z}}\right) .
$$

$\vec{z} Y$-periodic

$\vec{z}=\overrightarrow{0}$ on $a T$

$\operatorname{div} \vec{z}=0$ in $Y$

Notice that $\vec{z}_{\xi}^{*}$ depends linearly on $\xi$, that is : $\vec{z}_{\xi}^{*}=\sum_{k} \xi_{k} \vec{z}_{k}^{*}$, where $\vec{z}_{k}^{*}$ is the solution of (22) with $\xi=\vec{e}_{k}$.

From (17), one derives

$$
\begin{aligned}
& \frac{1}{2} \int_{Y \backslash \overline{a T}} \sum_{i j}\left(e_{i j}\left(\vec{z}_{\xi}\right)\right)^{2} d y+g \int_{Y \backslash \overline{a T}}\left(\sum_{i j}\left(e_{i j}\left(\vec{z}_{\xi}\right)\right)^{2}\right)^{1 / 2} d y-\xi \cdot \widetilde{\vec{z}}_{\xi} \leq \\
& \leq \frac{1}{2} \int_{Y \backslash \overline{a T}} \sum_{i j}\left(e_{i j}\left(\vec{z}_{\xi}^{*}\right)\right)^{2} d y+g \int_{Y \backslash \overline{a T}}\left(\sum_{i j}\left(e_{i j}\left(\vec{z}_{\xi}^{*}\right)\right)^{2}\right)^{1 / 2} d y-\xi \cdot \widetilde{\vec{z}}_{\xi}^{*} .
\end{aligned}
$$

Hence, from Lemma $3.4 \mathrm{~d}$ ), which has been proved by Lions and SanchezPalencia in [10], and from (22), one derives :

$$
\begin{aligned}
\frac{1}{2} \int_{Y \backslash \overline{a T}} \sum_{i j}\left(e_{i j}\left(\vec{z}_{\xi}\right)\right)^{2} d y \leq-\frac{1}{2} \int_{Y \backslash \overline{a T}} & \sum_{i j}\left(e_{i j}\left(\vec{z}_{\xi}^{*}\right)\right)^{2} d y+ \\
& +g \int_{Y \backslash \overline{a T}}\left(\sum_{i j}\left(e_{i j}\left(\vec{z}_{\xi}^{*}\right)\right)^{2}\right)^{1 / 2} d y
\end{aligned}
$$

And finally, using (21)

$$
A_{0} \xi \cdot \xi \geq \int_{Y \backslash \overline{a T}} \sum_{i j}\left(e_{i j}\left(\vec{z}_{\xi}^{*}\right)\right)^{2} d y-2 g \int_{Y \backslash \overline{a T}}\left(\sum_{i j}\left(e_{i j}\left(\vec{z}_{\xi}^{*}\right)\right)^{2}\right)^{1 / 2} d y .
$$

These three properties of $A_{0}$ prove that $A_{0}$ is maximal monotone and onto (see [3]), and Lemma $3.4 \mathrm{a}$ ) is proved.

Lemma $3.4 \mathrm{~b}$ ) is a consequence of (17), since $\widetilde{\vec{z}}_{\xi_{1}}=\widetilde{\vec{z}}_{\xi_{2}}$.

Lemma $3.4 \mathrm{c}$ ) is a consequence of (23) and following inequality which is deduced from (22) :

$$
\begin{aligned}
\frac{1}{2} \int_{Y \backslash \overline{a T}} \sum_{i j}\left(e_{i j}\left(\vec{z}_{\xi}^{*}\right)\right)^{2} d y-\xi \cdot \widetilde{\vec{z}}_{\xi} & \leq \frac{1}{2} \int_{Y \backslash \overline{a T}} \sum_{i j}\left(e_{i j}\left(\vec{z}_{\xi}\right)\right)^{2} d y-\xi \cdot \widetilde{\vec{z}}_{\xi} \\
& \leq \frac{1}{2} A_{0} \xi \cdot \xi-A_{0} \xi \cdot \xi \quad(\text { see }(21)) . \\
& -63-
\end{aligned}
$$




\section{A. Brillard}

\section{ACKNOWLEDGMENT}

Let me express my thanks to the referee who pointed to my attention the necessity of the discussion presented in the Remark 2.5.

\section{Références}

[1] Aтtouch (H.). - Variational convergence for functions and operators. Applicable Maths Series. Pitman (London) 1984 .

[2] Bensoussan (A.), Lions (J.L.), Papanicolaou (G.).- Asymptotic analysis for periodic structures. North Holland (Amsterdam) 1978.

[3] Brezis (H.). - Opérateurs maximaux monotones et semi-groupes de contraction dans les espaces de Hilbert. North Holland (Amsterdam) 1973.

[4] Brillard (A.). - Asymptotic behaviour of the solution of Mossolov and Miasnikov's problem in an open set with holes. Publications Univ. Haute Alsace $n^{\circ} 42$ 1986.

[5] Brillard (A.). - Asymptotic analysis of incompressible and viscous fluid flow through porous media. Brinkman's law via epi-convergence methods. Ann. Fac. Sci. Toulouse t. 8 Fasc. 2 p. 225-252 1987 and Publication Avamac Perpignan $n^{\circ} 85-111985$.

[6] De GIORGI (E.). - Convergence problems for functionals and operators. Proc. Int. Meeting on Recent methods in nonlinear analysis. Rome 1978. De Giorgi, Magenes, Mosco, Eds. Pitagora Ed. (Bologna) 1979.

[7] Duvaut (G.), Lions (J.L.). - Les inéquations en mécanique et physique, Dunod (Paris) 1972.

[8] Ekeland (I.), Temam (R.).- Analyse convexe et problèmes variationnels. Dunod (Paris) 1978.

[9] Levy (T.). - Loi de Darcy ou loi de Brinkman? CRAS Série II t. 2921981 p. 871-874.

[10] Lions (J.L.), SANChEz-PALENCia (E.). - Ecoulement d'un fluide viscoplastique de Bingham dans un milieu poreux. J. Maths. pures et appli. 601981 p. 341-360.

[11] Marchenko (A.V), Hruslov (E.Y.). - Boundary value problems in domains with close-grained boundaries. Naukova Dumka (Kiev) 1974 (in russian).

[12] SANChez-Palencia (E.).- Non-homogeneous media and vibration theory. Lecture Notes in Physics $n^{\circ} 127$. Springer Verlag (Berlin) 1980.

[13] Tartar (L.). - Incompressible fluid flow in a porous medium. Convergence of the homogenization process. Appendix in [12].

[14] Temam (R.). - Navier-Stokes equations. North Holland (Amsterdam) 1977. see also Deny-Lions Les espaces de type Beppo-Levy. Ann. Inst. Fourier. t. 5, 1954, p. 305-370, Necas J. Equations aux dérivées partielles. Presses de l'Université de Montreal. 1965 .

[15] Temam (R.). - Problèmes mathématiques en plasticité. Gauthier-Villars (Paris) 1983 . 